\title{
STAT3- and STAT5-dependent pathways competitively regulate the pan-differentiation of CD34pos cells into tumor-competent dendritic cells
}

\author{
Peter A. Cohen, ${ }^{1,2}$ Gary K. Koski, ${ }^{1,2}$ Brian J. Czerniecki, ${ }^{3}$ Kevin D. Bunting, ${ }^{2}$ Xin-Yuan Fu, ${ }^{4}$ Zhengqi Wang, ${ }^{2}$ Wen-Jun Zhang, ${ }^{4}$ \\ Charles S. Carter, ${ }^{5}$ Mohamed Awad, ${ }^{1}$ Christopher A. Distel, ${ }^{1}$ Hassan Nagem, ${ }^{1}$ Christopher C. Paustian, ${ }^{1}$ \\ Terrence D. Johnson, ${ }^{1}$ John F. Tisdale, ${ }^{6}$ and Suyu Shu ${ }^{1,2}$ \\ ${ }^{1}$ Center for Surgery Research, Cleveland Clinic Foundation/Lerner Research Institute, $\mathrm{OH} ;{ }^{2} \mathrm{Case}$ Comprehensive Cancer Center, Cleveland, OH; ${ }^{3}$ Department \\ of Surgery, University of Pennsylvania Medical Center, Philadelphia; ${ }^{4}$ Department of Microbiology and Immunology and the Walther Oncology Center, Indiana \\ University School of Medicine, Indianapolis; and ${ }^{5}$ Department of Transfusion Medicine and ${ }^{6}$ National Institute of Diabetes and Digestive and Kidney Diseases, \\ National Institutes of Health, Bethesda, MD
}

\begin{abstract}
The clinical outcomes of dendritic cell (DC)based immunotherapy remain disappointing, with DCs often displaying a tenuous capacity to complete maturation and DC1 polarization in the tumor host. Surprisingly, we observed that the capacity for successful DC1 polarization, including robust IL12p70 production, could be regulated by STAT-dependent events even prior to DC differentiation. Exposure of CD34 ${ }^{\text {pos }}$ cells to single-agent granulocyte-macrophage colony-stimulating factor (GMCSF) induced multilineage, STAT5-dependent differentiation, including DCs that failed to mature in
\end{abstract}

the absence of further exogenous signals. In contrast, Flt3L induced nearly global differentiation of CD34pos cells into spontaneously maturing DCs. IL-6 synergized with FIt3L to produce explosive, STAT3dependent proliferation of phenotypically undifferentiated cells that nevertheless functioned as committed DC1 precursors. Such precursors not only resisted many tumorassociated immunosuppressants, but also responded to tumor contact or TGF $\beta$ with facilitated DC maturation and IL12p70 production, and displayed a superior capacity to reverse tumor-induced T-cell tolerance.
GMCSF preempted Flt3L or Flt3L plus IL-6 licensing by blocking STAT3 activation and promoting STAT5-dependent differentiation. Paradoxically, following overt DC differentiation, STAT5 enhanced whereas STAT3 inhibited DC1 polarization. Therefore, nonoverlapping, sequential activation of STAT3 and STAT5, achievable by sequenced exposure to FIt3L plus IL-6, then GMCSF, selects for multilog expansion, programming, and DC1 polarization of tumor-competent DCs from CD34 ${ }^{\text {pos }}$ cells. (Blood. 2008;112:1832-1843)

\section{Introduction}

Dendritic cells (DCs) are the most potent antigen-presenting cells in the body and are used in many tumor vaccine immunotherapy trials, rarely with therapeutic impacts. ${ }^{1,2}$ DC preparations display a wide range of characteristics in vitro and in vivo, and it remains uncertain which individual properties may best promote successful immunotherapy. ${ }^{3-6}$

A variety of single agents, including CD40 ligand, Toll-like receptor (TLR) agonists, and calcium ionophore, can induce DC phenotypic maturation. ${ }^{3,4,6-9}$ Such maturation includes pronounced expression of MHC and costimulatory molecules, CD40 and CCR7, and IL- 8 secretion, but falls short of the DC's potential to achieve DC1 polarization, a highly effective state for promoting cell-mediated immunity. ${ }^{4,6,7,9,10} \mathrm{DC} 1$ polarization includes abundant production of IL12p70 heterodimer and IL-23, secretion of the chemokine MIP-1 $\beta$, and preferential expression of Delta- 4 Notch ligand. ${ }^{4,6,7}$ Such DC1 products are highly associated with chemoattraction and activation of T1type $\mathrm{CD}^{+}{ }^{+}$and $\mathrm{CD} 8{ }^{+}$T cells. ${ }^{4,6,7}$ Furthermore, IL12p70 production is critical to sensitize high-avidity $\mathrm{T}$ cells that directly recognize and kill tumor targets. $4,6,7$

Although desirable for antitumor immunity, DC1 polarization is more easily signaled by infectious agents than by tumor exposure. Immature DCs use recognition of pathogen- associated molecular patterns to assess the likelihood of host infection and the appropriateness of DC1 polarization. ${ }^{6,7}$ Individual TLRs signal DCs primarily through the MyD88 pathway (eg, TLR7-9) or TRIF pathway (eg, TLR3), with TLR4 evidencing pathway duplicity. ${ }^{7}$ Although activation of either pathway can induce elements of phenotypic DC maturation, dual pathway activation, or single pathway activation potentiated by exposure to IFN- $\gamma$ or CD40 ligation, is required for robust DC1 polarization. ${ }^{6,7}$

Fresh mobilization of $\mathrm{DC} 1$ precursors has the theoretic potential to promote cross-presentation of tumor Ags within the tumor host. Recent studies confirm the capacity of stem cell-mobilizing treatments, notably granulocyte-macrophage colony-stimulating factor (GMCSF), granulocyte colony-stimulating factor, fms-like tyrosine kinase receptor-3 (Flt3 ligand, Flt3L), or combined Flt3L plus GMCSF to mobilize DC precursors, ${ }^{5,11-13}$ but the potential of such precursors to achieve DC1 polarization is presently unclear. Flt3L plus GMCSF mobilization was recently reported to induce abundant infiltration of DCs into mouse tumors, but such DCs also activated regulatory $\mathrm{T}$ cells and promoted tumor tolerance. ${ }^{5}$ Immunosuppressive factors including IL-10, transforming growth factor beta (TGF- $\beta$ ), vascular endothelial growth factor (VEGF) and prostaglandin $\mathrm{E}_{2}\left(\mathrm{PGE}_{2}\right)$ are often produced by tumors, and
Submitted December 19, 2007; accepted May 22, 2008. Prepublished online as Blood First Edition paper, June 24, 2008; DOI 10.1182/blood-2007-12-130138.
The publication costs of this article were defrayed in part by page charge payment. Therefore, and solely to indicate this fact, this article is hereby marked "advertisement" in accordance with 18 USC section 1734. 
may impede mobilized DC precursors from attaining optimal maturation and DC1 polarization. ${ }^{14-17}$ However, mobilizing treatments themselves can also influence the later differentiation responses of stem cells..$^{5,11-13}$ We therefore postulated that particular stem cell proliferative treatments might provide conditioning signals that licensed rather than limited responsiveness to DC1 polarization stimuli.

Stem cell proliferation is inducible via multiple signaling pathways, including receptor-linked tyrosine kinases (Flt3L and stem cell factor $[\mathrm{SCF}]),{ }^{18-20} \mathrm{gp} 130$ (eg, IL-6), ${ }^{18,19}$ and the hematopoietin receptor superfamily (eg, GMCSF), ${ }^{20}$ During the systematic testing of such signaling agents we identified that combined exposure to Flt3L plus IL-6 (Flt3L + IL-6) not only synergized for stem cell proliferation, but also licensed CD34pos progenitor cells to forego multilineage differentiation in favor of STAT3-dependent, dedicated DC differentiation. Such STAT3-dependent DC differentiation proved highly conducive to DC1-type functional competence during later interactions with tumor. In contrast, exposure of CD34pos cells to GMCSF dominantly promoted an alternative, STAT5-dependent pathway of DC differentiation that was less conducive to tumor interactions.

\section{Methods}

\section{Mice and tumors}

All experiments were performed under institutionally approved animal research committee protocols adhering to USDA guidelines. Female $\mathrm{C} 57 \mathrm{BL} / 6 \mathrm{~N}, \mathrm{C} 3 \mathrm{H} / \mathrm{HeJ}$, and $\mathrm{BALB} / \mathrm{c}$ mice were purchased from Biologic Testing Branch, National Cancer Institute (NCI, Frederick, MD). They were maintained pathogen-free under UDSA guidelines and studied at 8 to 12 weeks or as indicated. MCA-203, -105, and -205 fibrosarcomas and B16 melanoma, syngeneic to C57BL/6N mice; CT26 colonic adenocarcinoma, syngeneic to BALB/c mice; and the 888 mel human melanoma line were maintained as described previously. ${ }^{21,22}$

After extensive C57BL/6N backcross, STAT5a/b knockout (KO) mice ${ }^{23}$ with hypomorphic STAT5 expression (N-terminal truncation ${ }^{24}$ ) were obtained by breeding heterozygotes to yield viable STAT5 $\mathrm{ab}^{-1-}$ pups. Mice were genotyped by polymerase chain reaction (PCR) of tail clip genomic DNA using primer pairs specific for STAT5a and/or STAT5b genes as previously described..$^{25}$

Following exhaustive C57BL/6N backcross, mice homozygous for a STAT3 allele with loxP sequences flanking critical exons 18 to 20 (F allele) were bred with Tie2-Cre mice (C allele) to generate mice with bone marrow (BM) conditionally knocked out for STAT3 (STAT3-CFF). ${ }^{26}$ Tail clip PCR genotyping distinguished a 490-bp product of wild-type STAT3 from the 520-bp STAT-F product, as well as the presence or absence of a Tie2-Cre 300-bp product.

\section{Reagents}

rhFlt3L (gift of Amgen, Thousand Oaks, CA); rhIL-2 (gift of Chiron, Emeryville, CA); rmGMCSF (gift of Immunex, Seattle, WA); rmCSF, rmIL-6, rmIL-10, rmVEGF, rmIL-3, rm thrombopoietin, rmIL-4, rmIFN- $\gamma$, rrhIL-7, and rhIL-15 (Peprotech, Rocky Hill, NJ); rhTGFß1 (R\&D, Minneapolis, MN); $\mathrm{PGE}_{2}$, LPS (Escherichia coli 026:B6), poly I:C, and prostaglandin $\mathrm{E}_{2}$ (Sigma-Aldrich, St Louis, MO); $\mathrm{CpG}$ (ODN 1826); and imiquimod (Invivogen, San Diego, CA) were used. Culture medium (CM) consisted of RPMI-1640 with $10 \%$ heatdeactivated FCS and conventional additives. ${ }^{21}$

\section{BM proliferation (step 1 of culture)}

Mouse BM suspensions were prepared from femurs and tibias. ${ }^{27}$ Following red blood cell (RBC) lysis by ammonium chloride, 12 to 15 million BM cells (CD34pos cell frequency 8\%-12\%) were cultured at 0.5 million/mL in $75-\mathrm{cm}^{2}$ flasks (Corning, Corning, $\mathrm{NY}$ ) at $10 \% \mathrm{CO}_{2}$ in
CM with the factors to be tested: hFlt3L $25 \mathrm{ng} / \mathrm{mL}$ (similar results $25-300 \mathrm{ng} / \mathrm{mL}$ ); mIL-6 $25 \mathrm{ng} / \mathrm{mL}$ (similar results $25-100 \mathrm{ng} / \mathrm{mL}$; $100 \mathrm{ng} / \mathrm{mL}$ rhIL-6 also effective); $\mathrm{mSCF} 25 \mathrm{ng} / \mathrm{mL}$ (similar results 25-100 ng/mL; rSCF also effective); rmGMCSF $10 \mathrm{ng} / \mathrm{mL}$ (similar results $10-25 \mathrm{ng} / \mathrm{mL}$ ); and $\mathrm{rmIL}-4 \quad 10 \mathrm{ng} / \mathrm{mL}$ (similar results 10-25 ng/mL). Recombinant thrombopoietin, IL-3, and fibronectin were also assessed, with negligible superimposed proliferative impacts (not shown). BM cells were normally cultured for 6 to 7 days, harvested, and washed twice in PBS before step 2 of culture.

\section{In vitro BM differentiation/maturation (step 2 of culture)}

Step 2 was initiated (0 hours) in CM, rmGMCSF, and optionally rmIL-4 in 24-well cluster plates, 4 million BM cells/well. DC1 polarization stimuli such as CpG (ODN1826, $5 \mu \mathrm{M}$ ) and lipopolysaccharide (LPS, $50 \mathrm{ng} / \mathrm{mL}$ ) were added between 18 to 24 hours, and cells harvested at 40 to 44 hours for analyses. When included, rmIL-10, rmVEGF, rhTGF $\beta 1$, or $\mathrm{PGE}_{2}$ was added between 0 to 24 hours of step 2 of culture. Alternatively, particulate tumor cells, either viable unirradiated, viable irradiated (10 $000 \mathrm{cGy}$ ), or killed freeze-thawed lysate, ${ }^{28}$ were added. Viable tumor cells were sometimes labeled with CFSE (Molecular Probes, Eugene, OR) to allow exclusion during fluorescence-activated cell sorting (FACS) analyses. ${ }^{21}$

\section{FACS analyses of cultured BM}

Cells were cultured in anti-CD32 mAb plus normal mouse IgG to block $\mathrm{FcR}$, then stained with fluorescently conjugated specific or isotype controls mAbs (BD-PharMingen, Mountain View, CA). ${ }^{29}$ When additionally assessing IL-12 production at the cellular level, the last 16 hours of step 2 of culture were performed in monensin (Golgistop; BD-PharMingen). Then, after FcR block and surface molecule staining, cells were treated with CytoPerm/CytoFix (BD-PharMingen), then stained with PE anti-mouse IL12p40 (BD-PharMingen) or PE isotype control.

Intracellular staining for TLR was performed directly on fixed, permeabilized cells with conjugated mAb (TLR3, TLR4, TLR8, TLR9), or indirectly with unconjugated mAb (TLR7), and appropriate controls from Imgenix (San Diego, CA). Staining for intracellular IRF4 and IRF8 was performed with reagents from Santa Cruz Biotechnology (Santa Cruz, CA) and mouse adsorbed $\mathrm{F}(\mathrm{ab})_{2}{ }_{2}$ fragments of donkey anti-goat $\mathrm{Ab}$ (Research Diagnostics, Concord, MA), following the method of Tamura et al..$^{30}$

To isolate CD34 $4^{\text {pos }}$ and CD34 $4^{\text {neg }}$ cells from freshly harvested BM, the latter were stained with FITC rat anti-mouse CD34 (dialyzed to remove sodium azide), then sorted on a FACSAria (BD Biosciences, San Jose, CA), yielding more than $96 \%$ pure $\mathrm{CD} 34^{\text {pos }}$ and $\mathrm{CD} 34^{\text {neg }}$ subpopulations.

In addition to PCR STAT determinations, pSTAT proteins were analyzed on the cellular level by FACS. ${ }^{31}$ Nuclei of cultured BM cells were permeabilized by sequential exposure to formalin (CytoFix) and 90\% methanol. Cells were then stained with PE- or FITC-conjugated anti-pSTAT3 (pY705), anti-pSTAT5 (pY694), or isotype controls (BD Biosciences).

\section{Enzyme-linked immunosorbent assays}

Supernatant contents of mIL12p70 heterodimer were quantified with BD-PharMingen reagents. mIFN- $\alpha$ and mIFN- $\beta$ were quantified with PBL Biomedical Laboratories kits (New Brunswick, NJ).

\section{T-cell cocultures}

Prior to T-cell harvest, DCs were prepared under various step 1 conditions in CM; step 2 was performed in $1 \%$ non-heat-deactivated mouse serum (MS) instead of FCS, during which BM cultures were exposed to viable irradiated tumor cells, then $\mathrm{CpG}$ plus LPS. On the day of DC harvest, T cells were freshly harvested from tumor-draining lymph nodes (TDLNs) of 12-day tumor-bearing mice. The L-selectin ${ }^{\text {low }}$ (tolerized pre-effector) fraction of $\mathrm{T}$ cells was isolated as previously described, ${ }^{29,32}$ and cultured in CM-MS with immobilized anti-CD3 ${ }^{29,32}$ or variously conditioned DCs. Beginning on day 2 of T-cell culture, some groups also received rhIL-2 (24 IU/mL), or rhIL-2, rhIL-7 
(50 ng/mL), and rhIL-15 ( $5 \mathrm{ng} / \mathrm{mL})$. T cells were harvested for assays and adoptive therapy after 12 days of culture.

\section{T-cell specificity assays (intracellular IFN- $\gamma$ )}

Cultured T cells were replated in fresh CM-MS at 2 million T cells/well in 24-well plates. Whole-cell irradiated tumor digests (5000 cGy) were added at 2 million cells/well as stimulators. Coculture proceeded for 18 hours, with monensin added the final 13 hours. At harvest, individual treatment groups were FcR blocked, then stained with FITC anti-CD4 and cychrome anti-CD8. Following fixation/permeabilization, cells were additionally stained with PE antimouse IFN- $\gamma$ or isotype controls, then analyzed.

\section{Adoptive immunotherapy}

Viable tumor cells (1.5 million) were injected into healthy syngeneic mice to establish intradermal tumors. ${ }^{29}$ Five or 10 days later, mice received conventional nonmyeloablative whole-body irradiation (WBI, $500 \mathrm{cGy}$ ), ${ }^{29}$ followed by culture-activated T cells intravenously. Perpendicular bidimensional tumor measurements were performed twice weekly. Mice were killed when bidimensional product exceeded $225 \mathrm{~mm}^{2}$.

\section{In vivo monitoring of $\mathrm{BM}$ cells}

Cultured BM cells were labeled with $\mathrm{CFSE}^{21}$ and injected intravenously into syngeneic mice bearing 10-day subcutaneous MCA-203 or MCA-105 tumors. Forty-eight hours later, mice were killed and tumors harvested and enzymatically digested to produce whole-cell digests, with spleen cell suspensions prepared in parallel. ${ }^{21}$ Preparations from individual mice were analyzed by FACS for CFSE $^{\text {pos }}$ cell frequencies. Groups were then costained with PE-conjugated $\mathrm{mAb}$ against DC-associated surface determinants, and FACS gated to analyze the CFSE ${ }^{\text {pos }}$ subpopulation.

\section{Statistics}

Survival among treatment groups was compared by Fisher exact test. Individual mice were scored for final treatment outcome (lethal tumor vs cure) and treatment groups compared. A 2-tailed $P$ value less than .05 was deemed significant. Proliferative synergy was assessed by Wilcoxon signed rank test for paired data (proliferation following exposure to combined factors vs summed synchronous proliferations of the individual factors). Trafficking accumulation of CFSE-labeled cells and FACS pSTAT quantitations for cultured BM were assessed by Wilcoxon signed rank test for paired data. In all cases, a 2-tailed $P$ value less than .05 was deemed significant.

\section{Results}

Later potentials for DC differentiation, DC maturation, and DC1 polarization are determined by early $C D 34^{\text {pos }}$ cell conditioning

We cultured fresh mouse BM in 2 steps, a 6- to 7-day proliferative culture (step 1) and a 48- to 72-hour postproliferative culture (step 2), followed in some experiments by T-cell coculture (Figure 1A).

Consistent with previous reports, ${ }^{18-20} 3$ treatment pairings (Flt3L + IL-6, SCF + IL-6, or Flt3L + GMCSF) produced synergistic proliferation during step 1 of culture (Figure 1B). Such expansions represented a selective 35- to 80-fold numeric expansion of the CD34pos cell subpopulation, with rapid dropout of initially CD34 ${ }^{\text {neg }}$ cells (Figure $1 C$ ). CD34pos cells continued brisk expansion for at least 1 additional week in culture if replenished with the same treatment pairings (not shown).

Even though Flt3L is myeloproliferative when administered to animals, ${ }^{5,33}$ step 1 of culture with single-agent Flt $3 \mathrm{~L}$ produced poor yields, even when dosing extended up to $300 \mathrm{ng} / \mathrm{mL}$ (Figure 1B and not shown). This validated previous reports that single-agent Flt3L is poorly proliferogenic ex vivo unless BM cultures are seeded at sufficiently high density to confer natural IL-6 supplementation. ${ }^{34}$

Prior to initial culture, freshly harvested CD34pos BM cells rarely displayed DC or other lineage markers (not shown). By the end of step 1, however, cultures treated with either single-agent Flt3L or single-agent GMCSF displayed frequent differentiation into immature DCs, based on their dual positivity for CD11c and MHC class II and low expression of CD40 and B7.2 (Figure 1D; also see Figure S1A for full tested panel, available on the Blood website; see the Supplemental Materials link at the top of the online article). Conventional DCs (positive for CD11bpos but negative for $\mathrm{B} 220^{\text {neg }}$ ) predominated in both instances (Figure S1B). Uniformly high MHC class II expression was a hallmark of Flt3Linduced DC differentiation, whereas GMCSF-induced DCs were heterogeneous and predominantly low in regard to MHC class II expression (Figure 1D).

Since Flt3L by itself was poorly proliferogenic (Figure 1B), we examined the superimposed impacts of IL-6 or GMCSF, since either agent produced proliferative synergy in conjunction with Flt3L (Figure 1B). BM proliferatively conditioned with Flt3L + IL-6 failed to acquire either CD11c or MHC class II expression, due to a pronounced antidifferentiative effect attributable to IL-6 (Figure 1D). In contrast, BM conditioned with either Flt3L + GMCSF or Flt3L + IL-6 + GMCSF developed heterogeneous differentiation that closely resembled treatment with GMCSF alone (Figure 1D). Therefore, even though IL-6 could exert a pronounced antidifferentiative effort upon Flt3L cultures, GMCSF could dominantly antagonize the conditioning impacts of both Flt3L and IL-6 during step 1 of culture.

Following step 1 conditioning, step 2 cultures were exposed to TLR agonists to examine their real-time potentials for DC1 polarization (Figure 1E). ${ }^{6,7}$ Since both TLR9 and TLR4 were invariably expressed at the end of step 1 mouse BM cultures (not shown), we standardly used CpG (ODN 1826) and lipopolysaccharide (LPS) during step 2 of culture to elicit coordinate activation of MyD88 and TRIF pathways. ${ }^{6,7}$

Step 1 conditioning with single-agent GMCSF rendered a large proportion of cells hyporesponsive or unresponsive to subsequent TLR agonists, as evidenced by limited or absent up-regulation of MHC class II, CD40, and B7.2 expression (Figure 1E; Figure $\mathrm{S} 2 \mathrm{~A}$ ). Gr-1 coexpression was a common feature of poorly responsive subpopulation(s) (Figure S2B). ${ }^{35,36}$

In contrast, step 1 conditioning with single-agent Flt3L licensed consistently high TLR responsiveness, manifested by nearly global phenotypic DC maturation and only scant Gr-1 ${ }^{\text {pos }}$ elements during subsequent step 2 of culture (Figure 1E; Figure S2B). However, Flt3L's global licensing impacts were antagonized if GMCSF was also included during step 1 of culture (Figure 1E; Figure S2A). Such inhibition was not observed if initial exposure to GMCSF was deferred until step 2 of culture (not shown).

In contrast to GMCSF, IL-6 not only produced proliferative synergy in conjunction with Flt3L (Figure 1B,C), but also promoted Flt3L's global licensing impacts. Although an undifferentiated state persisted during step 1 conditioning in Flt3L + IL-6 (Figure 1D), nearly the entire expanded CD34pos progenitor cell pool responded to subsequent TLR stimulation with DC differentiation, robust phenotypic maturation, and nearly uniform IL-12 production (Figures 1E; S2A). IFN- $\beta$ was coelicited rather than IFN- $\alpha$ by all tested TLR agonist combinations, indicating that conventional DC differentiation dominated during step 2 of culture (Figure S2C). ${ }^{7}$ However, as for Flt3L, Flt3L + IL-6's global licensing impacts were abrogated if GMCSF was also included during step 1 of culture (Figure 1E; Figure S2A). 
A

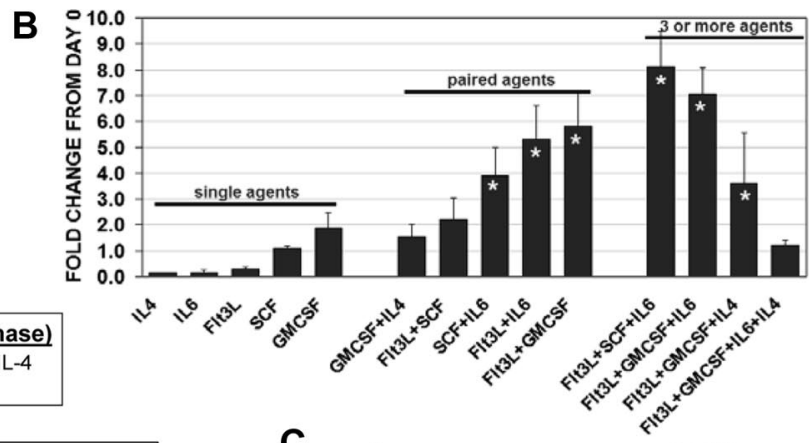

Step 1 BM Culture (Proliferative phase) Harvest 6-7 days later

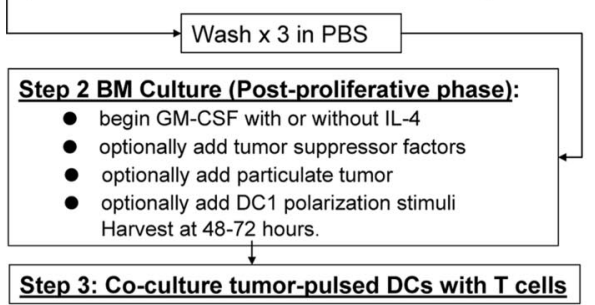

C
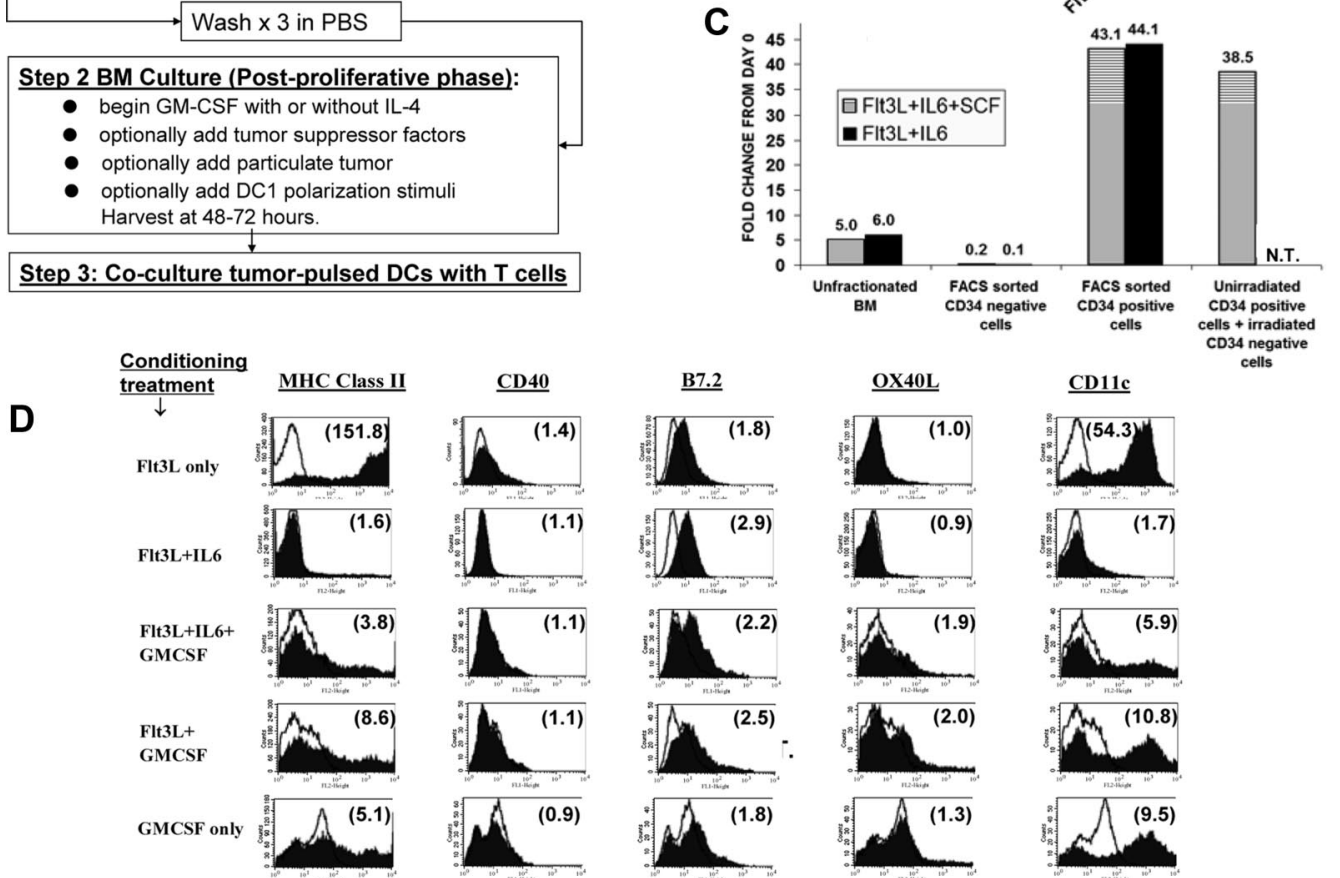

$\underline{\text { CD40 }}$

$\underline{B 7.2}$

$\underline{\mathrm{OX} 40 \mathrm{~L}}$
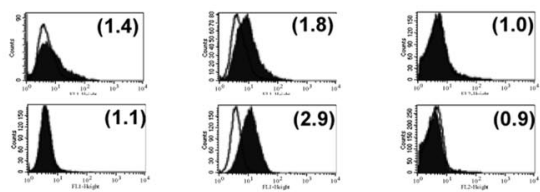

$\underline{\text { CD11c }}$
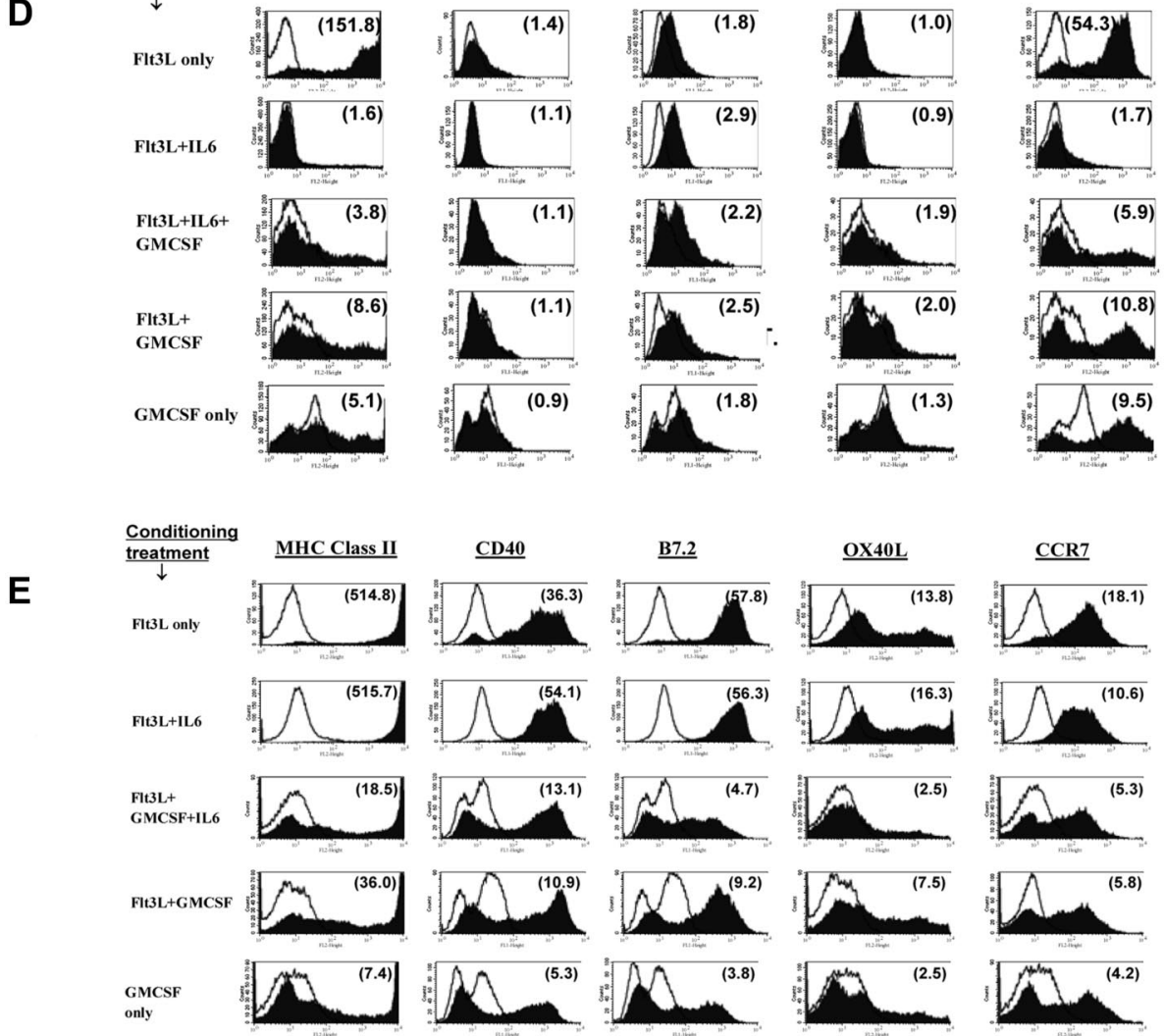

Figure 1. Numeric expansion and differentiation following exposure of bone marrow (BM) to various factors. (A) Schematic of test culture system. (B) BM freshly harvested from C57BL/6 mice was cultured for 6 days (step 1 culture) in the specified factors (dosing in "Methods"), then counted. Each bar represents averaged 3 to 11 determinations plus or minus SD, each performed in synchronous comparison to at least five other groups. Asterisked bars indicate treatment combinations that displayed significant proliferative synergy (see "Methods"). (C) Fresh uncultured mouse BM cell suspensions were mAb-stained and FACS sorted to separate CD34 ${ }^{+}$and CD34subpopulations. CD34+ cells initially represented $10.8 \%( \pm 7 \%)$ of the total BM cells. The following groups were then subjected to 6 -day step 1 culture either in Flt3L + SCF plus IL-6 (striped bars) or in Flt3L + IL-6 (solid bars): unfractionated BM (12-15 million per flask); FACS sorted CD34- cells (12-15 million per flask); FACS-sorted CD34+ cells (2-3 million per flask); or FACS-sorted, unirradiated CD34+ cells plus irradiated (3000 cGy) CD34- cells. Labels above bars indicate fold numeric expansion during the 6-day culture. All numeric expansion observed was attributable to proliferation of the $\mathrm{CD}_{3} 4^{+}$subpopulation, with no $\mathrm{CD} 34^{-}$feeder layer requirement. (N.T. indicates condition not tested). (D) Surface expression profiles at end of 6-day step 1 culture in various conditioning treatments. Individual treatments are listed in far left column. Ten different treatments were synchronously compared (all groups shown in Figure S1A). Number within each histogram plot indicates the mean fluorescence specificity index for the molecule tested, defined as the geomean fluorescent intensity of all cells after staining with the specified mAb (filled histogram), divided by the geomean background staining intensity for isotype control mAb (unfilled histogram). Results shown are representative of three comprehensive comparisons. (E) Following the step 1 conditioning treatments listed in far left column, each group was replated for step 2 in fresh medium with GMCSF plus IL-4 for 24 hours, followed by overnight exposure to paired TLR agonists (CpG ODN 1826 and LPS). FACS analyses were then performed on day 2 of this step 2 culture. Ten different conditioning treatments were compared (selected groups shown in pane E; all groups shown in Figure S2A). Far right column shows gross numeric expansion during initial step 1 culture. Number within each histogram is the calculated mean fluorescence specificity index (see panel D). Representative of three comprehensive comparison experiments. 
A

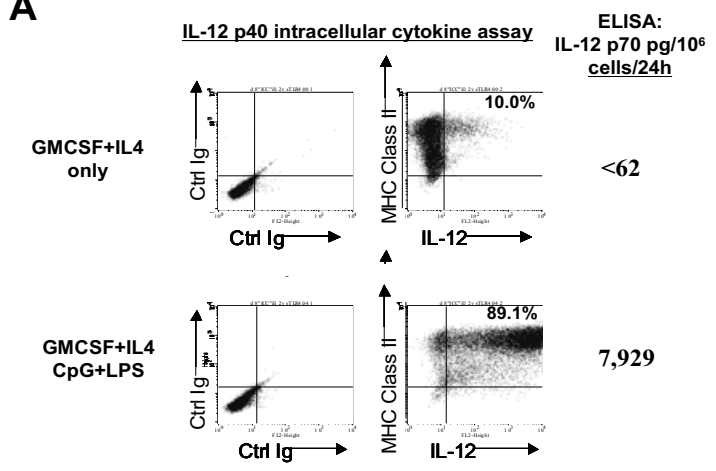

C

Step 2

$\frac{\frac{\text { treatment }}{\downarrow}}{\downarrow}$

Normal activation
$(0 \mathrm{hr}$ IL4+GMCSF, $24 \mathrm{~h} \mathrm{CpG}$, LPS

Normal activation plus $0 \mathrm{hr}$ mIL-10 $25 \mathrm{ng} / \mathrm{ml}$

Normal activation
plus $0 \mathrm{hr}$ hTGF $\beta 1$ plus $0 \mathrm{hr}$ hTGF $\beta 1$ Normal activation
plus $0 \mathrm{hr} \mathrm{mVEGF}$ $50 \mathrm{ng} / \mathrm{ml}$

Normal activation plus $0 \mathrm{hr} \mathrm{PGE}_{2} 4$ $\mu \mathrm{g} / \mathrm{ml}$

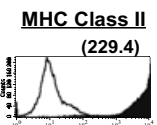

(262.5)
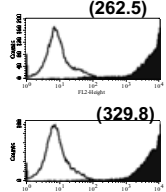

(283.2)
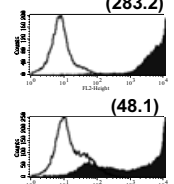

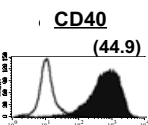

(63.7)
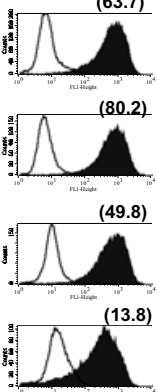

B7.2

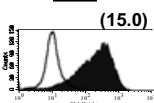

(17.2)
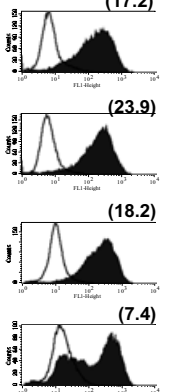

B

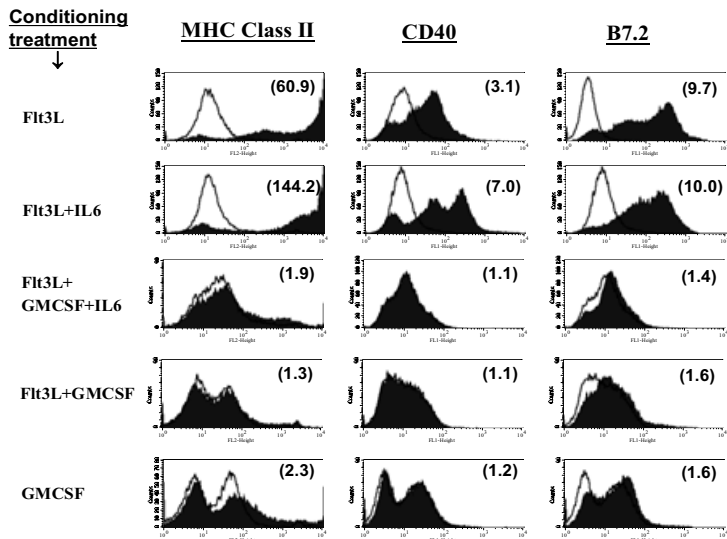

D

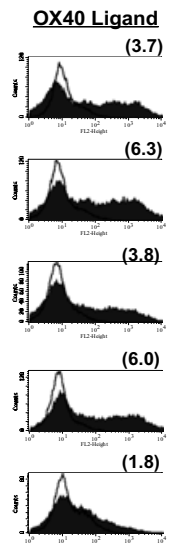

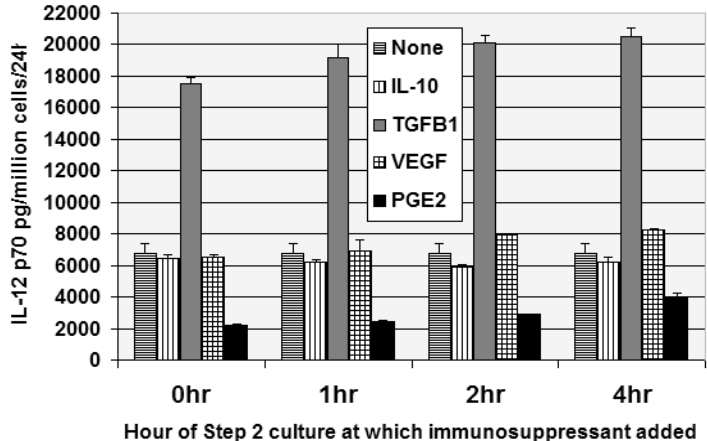

Hour of Step 2 culture at which immunosuppressant added

Figure 2. Stem cells conditioned in FIt3L + IL-6 are licensed for DC1-polarization, spontaneous maturation, and resistance to tumor-associated immunosuppressive factors. (A) Production of IL-12 by Flt3L + IL-6-conditioned BM cells following step 2 exposure to CpG plus LPS. Culture was performed as in Figure 1D, comparing outcomes with or without CpG plus LPS treatment. FACS dot plots show results of intracellular cytokine assays for IL12p40 production. Percentages shown in upper right quadrants are those of total BM cells specifically staining dually positive for MHC class II and intracellular IL-12 at the end of culture. Numbers in far right column show ELISA content of IL12p70 heterodimer from culture supernatants run in parallel without monensin. Data are representative of 8 experiments. (B) Step 2 culture as in Figure 1E, except following step 1 conditioning each group was replated in fresh medium for 72 hours solely with rGMCSF (no IL-4 or TLR agonists), after which FACS analyses were performed (selected groups shown in panel B; all groups shown in Figure S3a). Number within each histogram is the calculated mean fluorescence specificity index at 72 hours. Results shown are representative of 3 comprehensive comparisons. A similar but even more pronounced pattern of spontaneous maturation was observed during 48 to 72 hours step 2 culture in GMCSF + IL-4 (Figure S3B). (C) Step 2 cultures performed as in Figure 1E except that tumor-associated immunosuppressive factors were also added at 0 hour as listed in far left column. Data are representative of 3 experiments. (D) Same as in panel A, except listed immunosuppressive factors were added at 0 hour, 1 hour, 2 hours, or 4 hours of step 2 culture, with supernatants analyzed by ELISA in triplicate for IL12p70 content at 44 hours of step 2 culture. Error bars indicate SD. Data are representative of 3 experiments.

The functional consequences of each of these conditioning regimens proved age independent, with indistinguishable outcomes observed for BM obtained from mice aged 4 to 80 weeks (not shown). Similar responses were observed in all tested mouse strains.

\section{Prior Flt3L or Flt3L + IL-6 conditioning promotes spontaneous DC maturation and consistent TLR/IRF expression}

Following lineage commitment, DCs typically remain immature unless they are exposed to exogenous signals such as CD40 ligation, calcium ionophore, or TLR agonists.,4,6-9 It was observed, however, that BM cells conditioned in single-agent Flt3L subsequently displayed spontaneous DC maturation, even when GMCSF was the only exogenous supplement provided during step 2 of culture (Figure 2B; Figure S3A). Similar spontaneous DC maturation was also observed following step 1 proliferative conditioning in Flt3L + IL- 6 , even though the inclusion of IL-6 had delayed the onset of DC differentiation until the onset of step 2 of culture (Figure 1D vs Figure 2B; Figure S3A). Spontaneous DC maturation following either
Flt3L or Flt3L + IL-6 conditioning was even more vigorous when IL-4 was also provided during step 2 of culture (Figure $\mathrm{S} 3 \mathrm{~B}$ ). Marked up-regulation of the endocytic C-type lectin receptor DEC-205 was a conspicuous component of such spontaneous maturation (Figure S3C).

For all conditioning treatments other than Flt3L or Flt3L + IL-6, spontaneous step 2 maturation was either highly attenuated or not observed. Importantly, Flt3L + IL-6 was the only factor pairing that resulted both in proliferative synergy during step 1 of culture and in spontaneous DC maturation during step 2 of culture (Figure 2B; Figure S3). Even when other conditioning treatments evoked limited elements of DC maturation during step 1 of culture (Figure 1D; Figure S1A), such elements spontaneously reverted during step 2 of culture unless exogenous maturational stimuli such as TLR agonists were also provided (Figures 1E vs 2B; full panels in Figures S2A vs S3). Flt3L or Flt3L + IL-6's capacity to license spontaneous DC maturation was abrogated, however, if GMCSF was also included during step 1 conditioning (Figure 2B; Figure S3). 
We characterized expression elements of the conventional DCs that dominated step 2 of culture following Flt3L + IL- 6 conditioning. Expression of TLR3, TLR4, TLR7, TLR8, and TLR9 remained uniformly detectable at all stages of culture, functionally confirmed by these cells' broad responsiveness to respective TLR agonists (Figures S2C and S4A). Interferon-regulatory factors IRF4 and IRF8 were dually expressed both by DC precursors and polarized DC1 (Figure S4B), demonstrating a largely homogeneous sequence of differentiation, maturation, and polarization following Flt3L + IL-6 conditioning.

\section{FIt3L + IL-6-conditioned DC precursors are stimulated rather than inhibited by tumor interactions}

We investigated whether Flt3L + IL-6 proliferative conditioning licensed uniform responsiveness to $\mathrm{DC} 1$ polarization stimuli even in the presence of putative immunosuppressive factors. We added factors at doses that equaled and exceeded those reported to inhibit the maturation of other DC preparations. ${ }^{14-17} \mathrm{IL}-10$ and VEGF exposure had negligible impacts upon DC1 polarization, whereas TGF $\beta 1$ exposure paradoxically enhanced both phenotypic maturation and IL-12 secretion (Figure 2C,D). Only early PGE 2 exposure detectably inhibited TLR agonistinduced phenotypic DC maturation and IL-12 production (Figure 2C,D). Nonetheless, a large subpopulation of Flt3L + IL-6-conditioned $\mathrm{BM}$ cells resisted $\mathrm{PGE}_{2}$ inhibition even at the beginning of step 2 of culture (Figure 2C, note the bimodal B7.2 expression), and such resistance became increasingly prevalent within hours of step 2 of culture (Figure 2D; Figure S5).

We tested the impact of exposing DCs to voluminous tumor burdens at 16 to 24 hours of step 2 of culture. To each well containing 4 million preconditioned BM cells, we added either 4 million freeze-thawed (killed) tumor cells; 3 million irradiated (10 $000 \mathrm{cGy}$ ), trypan-excluding apoptotic tumor bodies; or 2 million unirradiated, actively proliferating tumor cells.

After step 1 of Flt3L + IL-6 conditioning, contact with any of these tumor materials accelerated DC phenotypic maturation, mimicking the stimulatory impact of exposure to a single TLR agonist (Figure 3A). ${ }^{6,7}$ Combining such tumor exposure with IFN- $\gamma$ treatment induced IL12p70 production (Figure 3A), mimicking the impact of combined exposure to IFN- $\gamma$ plus a single TLR agonist. ${ }^{4,7}$ When other step 1 conditioning conditions were compared, activating effects of tumor were either highly attenuated or completely absent (Figure 3A).

The capacity of Flt3L + IL-6-conditioned DCs to be activated by tumor contact proved strain independent, occurring even for BALB/c BM-derived DCs despite this strain's Th2-biasing tendency $^{37}$ (not shown). All tested tumor lines stimulated DC maturation after Flt3L + IL-6 conditioning, including MCA-205 and MCA-203 sarcomas and B16 melanoma derived from C57BL/6N mice, CT26 colon adenocarcinoma derived from BALB/c mice, and $888 \mathrm{mel}$ from a melanoma patient, and was not attributable to endotoxin content (not shown). Both freshly harvested whole-cell tumor digests and established tumor lines proved stimulatory, indicating that host stromal cells were unessential, and DC maturation was stimulated whether tumor was syngeneic, allogeneic, or xenogeneic (not shown). Although fully killed lysate was effective, viable tumor proved more effective. Sequestration of tumor from Flt3L + IL-6-conditioned DC precursors by Transwell membranes (Corning) abrogated the activating effects of tumor, underscoring a requirement for direct contact; in contrast, phagocytosis of latex beads did not accelerate maturation (not shown).

\section{Flt3L + IL-6-conditioned DCs promote superior reversal of tumor-induced T-cell tolerance}

We examined the capacities of previously conditioned DCs to reverse tolerance in $\mathrm{T}$ cells harvested from mice bearing advanced tumors. L-selectin ${ }^{\text {low }} \mathrm{T}$ cells from tumor-draining lymph nodes (TDLNs) are naturally sensitized to the relevant tumor but are also tolerized, consequent to the progressive upstream tumor burden. ${ }^{29}$ In vitro exposure to anti-CD3, followed by IL-2 stimulation, can reverse tolerance and numerically expand antitumor effector T cells. ${ }^{29}$ However, such polyclonal stimulation causes $\mathrm{CD}^{+} \mathrm{T}$ cells to overgrow $\mathrm{CD} 4^{+}$ $\mathrm{T}$ cells, and lacks the element of antigen presentation needed to selectively promote the tumor-specific T-cell subset. ${ }^{29,32}$

Conditioned DC precursors were transferred to step 2 of culture, exposed to viable irradiated tumor, optionally further activated with CpG plus LPS, then cocultured with tolerized TDLN T cells from mice bearing the relevant tumor. Flt3L + IL-6-conditioned DCs efficiently reversed tolerance and stimulated robust T-cell proliferation, even when exogenous cytokines such as IL-2 were not added to coculture (Figures 3B and 4A). CpG plus LPS treatment enhanced but was unessential for such efficacy (not shown). In contrast, DCs prepared after other conditioning treatments typically proved lethal to $\mathrm{T}$ cells, and toxicity could not be prevented by exogenous IL-2, IL-15, and/or IL-7 (Figures 3B and 4A). Cocultures driven by Flt3L + IL-6-conditioned DCs displayed superior outgrowth of both $\mathrm{CD} 4^{+}$and $\mathrm{CD} 8^{+}$tumor-specific T cells compared with anti-CD3 treatment (Figure 4Bi,ii), and were highly potent when provided as adoptive therapy against early or advanced established tumors (Figure 4C; Figure S6).

\section{FIt3L + IL-6-conditioned CD34pos cells achieve spontaneous intratumoral DC maturation in vivo}

Following various step 1 culture treatments, BM cells were CFSE labeled and administered to 10-day tumor-bearing mice. Cells injected immediately after step 1 conditioning with Flt3L + GMCSF or Flt3L + GMCSF + IL-6 displayed negligible trafficking into either tumor or spleen. In contrast, Flt3L plus IL-6-conditioned BM cells infiltrated both established tumors and spleen, achieving essentially uniform DC differentiation at either location (Figure 5A,B). Moreover, accelerated DC maturation was observed following entry into tumor compared with spleen (Figure 5B), consistent with observed stimulatory impacts of tumor contact in vitro during step 2 of culture (Figure $3 \mathrm{~A}$ ).

\section{FIt3L- versus GMCSF-conditioned DC programming reflects competing STAT3- versus STAT5-dependent events}

Knockout studies have demonstrated that Flt3 ligation transitions CD34pos common precursors into committed DC precursors via a STAT3-dependent process, whereas GMCSF promotes STAT3independent myeloid differentiation. ${ }^{38}$ IL-6 signaling is known to induce gp130-mediated STAT3 activation in CD34 ${ }^{\text {pos }}$ cells, ${ }^{39}$ whereas GMCSF induces more complex STAT modulations, including STAT5 activation, at several stages of myeloid differentiation. ${ }^{38,40,41}$ Because step 1 GMCSF consistently abrogated the unique conditioning impacts of Flt3L or Flt3L + IL-6, we examined whether STAT modulations played a pivotal role in GMCSF's apparently dominant regulation.

Step 1 IL-6 in the absence of GMCSF produced sustained activation of STAT3 but not STAT5, as evidenced by intranuclear staining for pSTAT3 (pY705) and pSTAT5 (pY694) (Figure 6A end step 1; Figure S7). In contrast, inclusion of GMCSF as a 
A

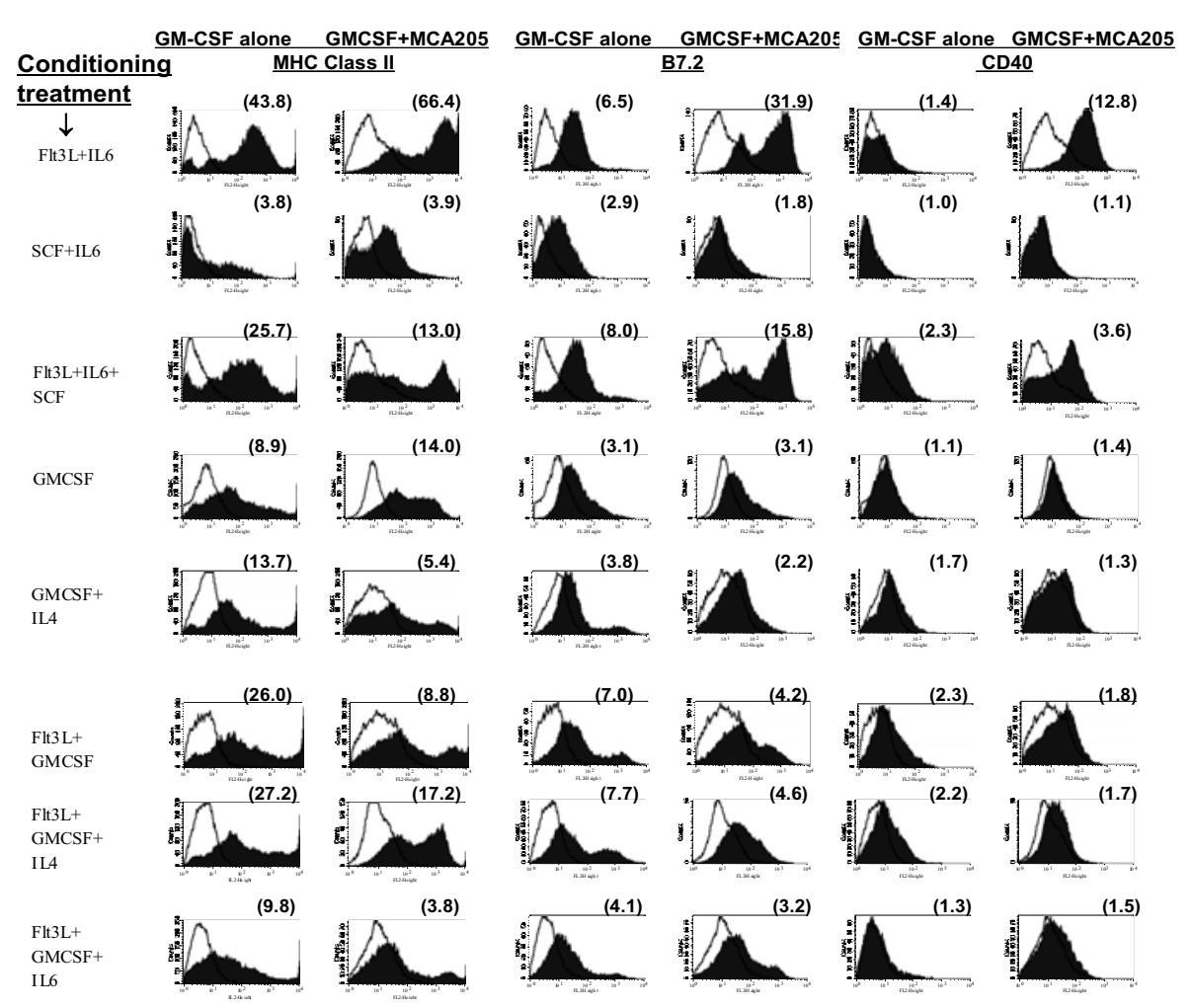

IL-2 70 production when IFN- $\gamma$ also added with MCA-205

(pg/24h/106 cells:)

886

191

186

159

112

191

\section{B}
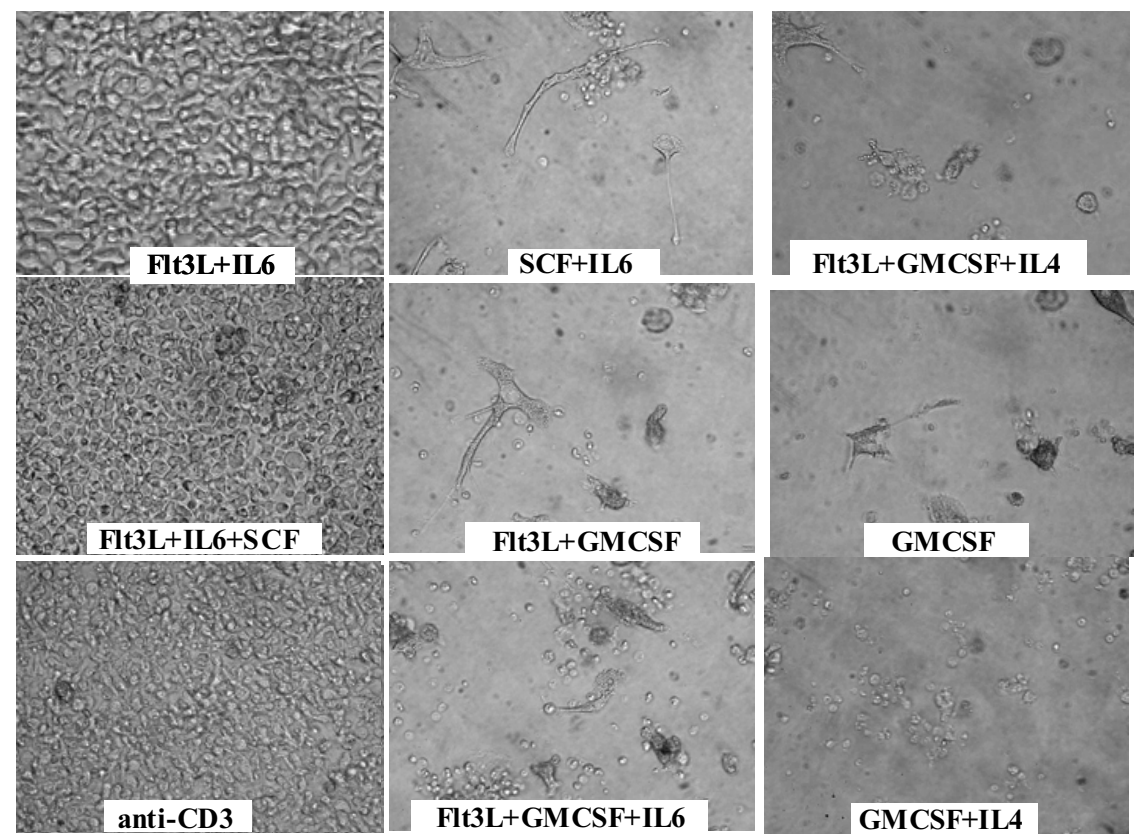

Figure 3. Impact of tumor on conditioned BM cells, and impact of tumor-pulsed DCs upon subsequent T-cell cocultures. (A) Following step 1 conditioning treatments listed in far left column, each group was replated at 4 million cells/well in fresh medium with only GMCSF added at the beginning of step 2 culture. Twenty-four hours later, individual wells were also exposed to 3 million viable irradiated MCA-205 tumor cells. FACS analyses of cells were performed at 44 hours (20 hours after addition of tumor). Histograms display expression of MHC class II, CD40, and B7.2 for each conditioning treatment, with or without exposure to tumor. The far right column displays supernatant content of IL12p70 heterodimer 20 hours after tumor exposure when rmIFN- $\gamma$ was also added to culture. Supernatant IL12p70 content was below detection ( $<31 \mathrm{pg} / 24 \mathrm{hr})$ following exposure to either rmIFN- $\gamma$ or to tumor alone (not shown). Data are representative of 3 full comparison experiments. (B) Photos of individual T-cell cultures after 6-day coculture with tumor-pulsed DCs or with anti-CD3. Labels denote step 1 DC conditioning treatments; step 2 DC cultures were performed in rGMCSF + rlL-4, with irradiated MCA-203 cells added at 24 hours and CpG + LPS added at 44 hours. Four hours later, DCs were harvested for coculture with L-selectinlow T cells freshly harvested from MCA-203 tumor-bearing mice. T cells were cocultured with DCs at a 8:1 ratio or were activated with immobilized anti-CD3. Cocultures shown also received exogenous IL-2 + IL-7 + IL-15. Data are representative of 3 full comparison experiments, using an Olympus IX50 inverted microscope, an Olympus CPlan $10 \times / 0.25$ PhC objective lens, a Sony DSC-S85 Cybershot, and Adobe Photoshop (Adobe Systems, Mountain View, CA) for compilation. 

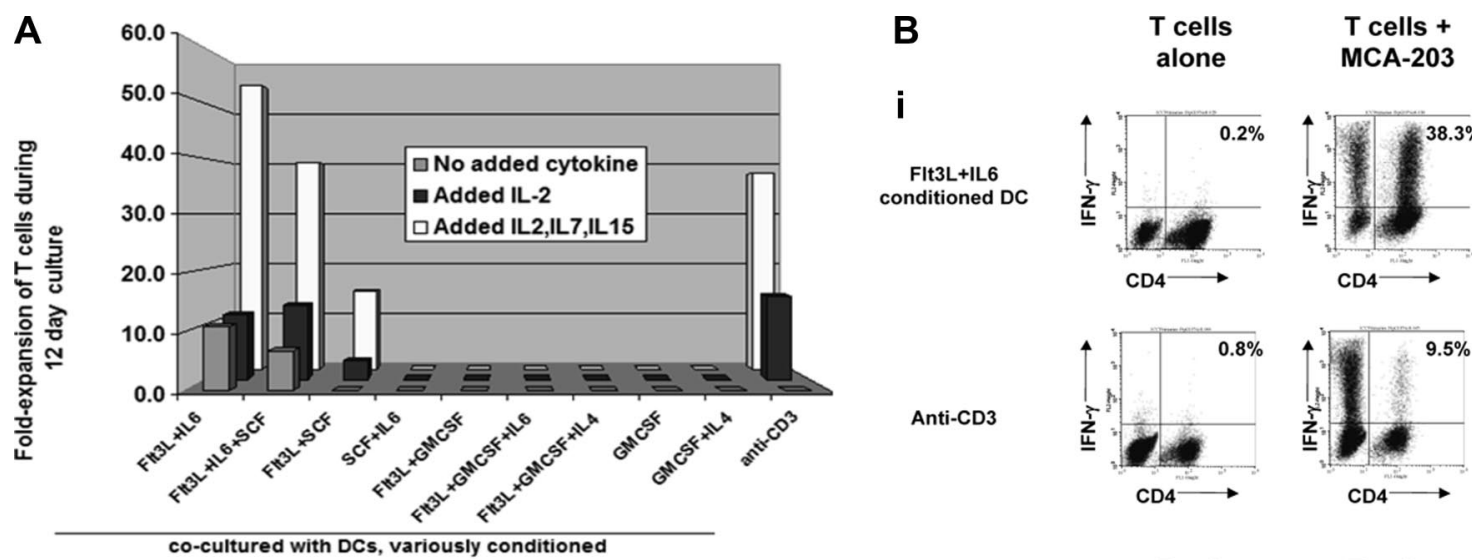

T cells + MCA-105

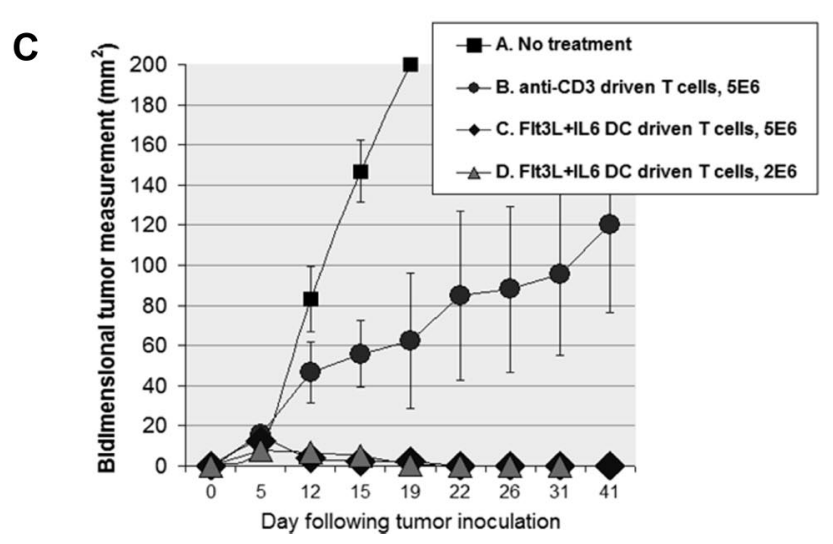

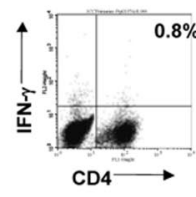

T cells
alone

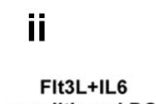
conditioned DC

Anti-CD3
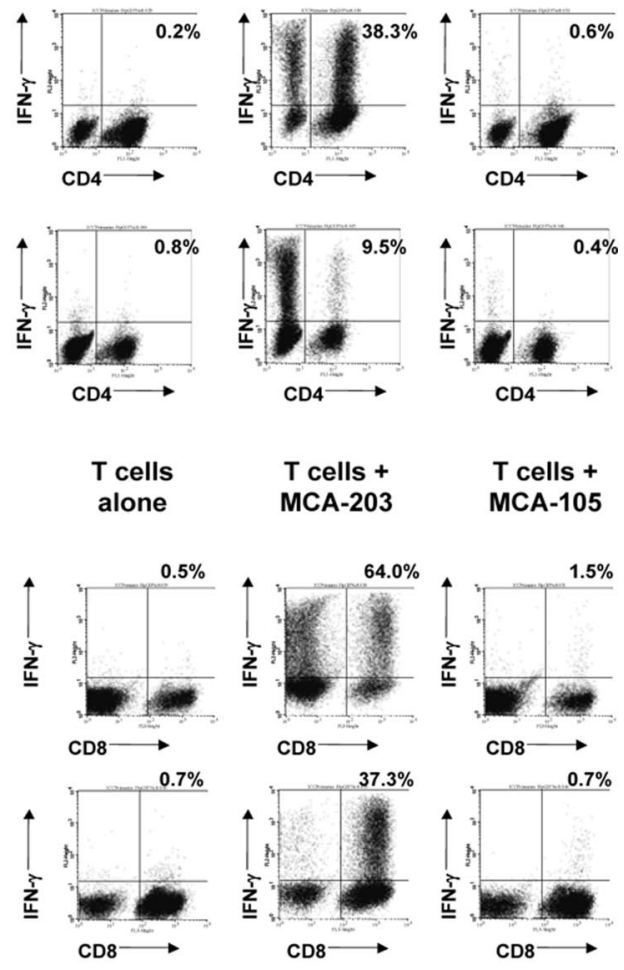

T cells + MCA-203

T cells +
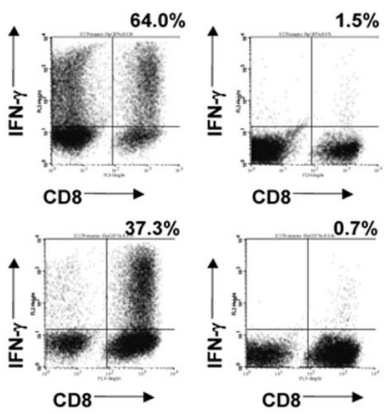

Figure 4. Impacts of initial step $1 \mathrm{DC}$ conditioning treatments upon subsequent culture of T cells from tumor-bearing mice. Tumor-pulsed DC preparations and co-cultures were performed as in Figure 3B. (A) T-cell fold-expansion 12 days after initial exposure to tumor-pulsed DCs or to anti-CD3; labels indicate prior DC conditioning treatments. As distinguished in the legend, the displayed T-cell expansions were performed either with no added cytokine, added rIL-2 only (24 IU/mL beginning day 2 of T-cell culture), or IL-2 + IL-7 + IL-15 (50 ng IL-7, 5 ng IL-15). Data are representative of 3 experiments. (B) Coculture of T cells from tumor-bearing mice with Flt3L + IL-6conditioned DCs results in superior expansion of tumor-specific T cells. Step $1 \mathrm{DC}$ conditioning was performed with Flt3L + IL-6, rest of DC preparation and T-cell cocultures as in Figure 3B. After a 12-day coculture, T cells were harvested and replated either alone or with irradiated MCA-203 or MCA-105 as stimulator cells. Monensin (Golgistop) was added after 5 hours, and cells analyzed after an additional 12 hours for intracellular IFN $\gamma$ production. Dot plots show T cells recultured after expansion in IL-2 + IL-7 + IL-15, but coculture with tumor pulsed, Flt3L + IL-6-conditioned DCs yielded virtually identical tumor-specificity even when no cytokines were added during the 12-day coculture (not shown). (Bi) Dot plots display CD4 versus IFN $\gamma$ staining; the percentages shown in each dot plot is that of total CD4 cells producing IFN $\gamma$. (Bii) Dot plots display CD8 versus IFN $\gamma$ staining; the percentages in each dotplot is that of total CD8 cells producing IFN $\gamma$. This is representative of 6 experiments. (C) T cells driven with Flt3L + IL-6-conditioned DCs are highly effective as adoptive therapy. Five-day established MCA-203 subcutaneous tumors were treated intravenously with T cells from MCA-203 bearers after 12-day culture driven by Flt3L + IL-6-conditioned, tumor-pulsed DCs or by anti-CD3. Conventional nonmyelablative total body irradiation ( 500 cGy) was given as an adjunct prior to T cells. Cure rates were 0/5 (A, No treatment); 2/5 (B, 5 million anti-CD3 driven T cells); $5 / 5$ (C, 5 million Flt3L + IL-6 DC-driven T cells); $5 / 5$ (D, same as $C$ but 2 million T cells). Treatment outcome $A$ versus $C / D, P<.008 ; A$ versus $B, P<.141 ; B$ versus $C / D, P=.04$. T-cell cultures driven by Flt3L + IL-6-conditioned DCs were also highly effective against more advanced tumors (Figure S6). Data are representative of 4 experiments. Error bars represent SD.

component of any step 1 regimen depressed STAT3 activation and produced biphasic up-regulation of pSTAT5 (Figure 6A end step 1; Figure S7). These distinctive pSTAT expression patterns modulated again during step 2 of culture, however, when cultures previously conditioned in Flt3L + IL-6 now displayed the greatest capacity for STAT5 activation (Figure 6A end step 2).

We next examined how STAT knockout BM preparations responded to Flt3L + IL-6 conditioning. Consistent with STAT3's putative obligate role in Flt3L-induced DC differentiation, ${ }^{38}$ we observed that STAT3 ${ }^{\text {KO }}$ BM could not survive Flt3L + IL-6 in vitro conditioning (not shown). In contrast, STAT5 ${ }^{\mathrm{KO}} \mathrm{BM}$ responded to Flt3L + IL-6 conditioning with intact robust proliferation (not shown) and nearly uniform DC differentiation during subsequent step 2 of culture (Figure 6B row 2). Nonetheless, compared with wild-type littermates, Flt3L + IL-6-conditioned STAT5 $^{\text {KO }}$ DCs displayed submaximal DC maturation and IL12p70 production (Figure 6B row 2 vs row 1 and Figure 6D left). Therefore, the proliferative and differentiative impacts of Flt3L + IL-6 conditioning were absolutely STAT3-dependent, whereas subsequent phenotypic maturation and $\mathrm{DC} 1$ polarization were at least partially STAT5-dependent.

We also examined how STAT ${ }^{\mathrm{KO}}$ BM preparations responded to GMCSF-containing step 1 regimens (GMCSF alone, Flt3L + GMCSF, or Flt3L + GMCSF + IL-6). All of these GMCSF-based conditioning regimens were strikingly ineffective for generating DCs from STAT5 ${ }^{\mathrm{KO}} \mathrm{BM}$, instead yielding predominantly Gr-1 ${ }^{\text {pos }}$, MHC class II ${ }^{\text {neg }}$ cells that displayed morphologic features of mature neutrophils (eg, Figure 6B row 4, and data not shown). In contrast, STAT3 ${ }^{\mathrm{KO}}$ BM displayed normal proliferative kinetics (not shown) and typical heterogeneous differentiation, including DC differentiation, in response to all GMCSF-based regimens (Figure 6C row 4). Following GMCSF-based conditioning, however, STAT3 ${ }^{\text {KO }}$ DCs displayed an abnormally heightened maturational and IL12p70 response to TLR stimulation (Figure 6C row 4 vs row 3 and Figure $6 \mathrm{D}$ right). Therefore, all tested GMCSF-containing regimens required intact STAT5 for DC differentiation, and later phenotypic maturation and DC1 polarization displayed the capacity for negative regulation through STAT3. 
A

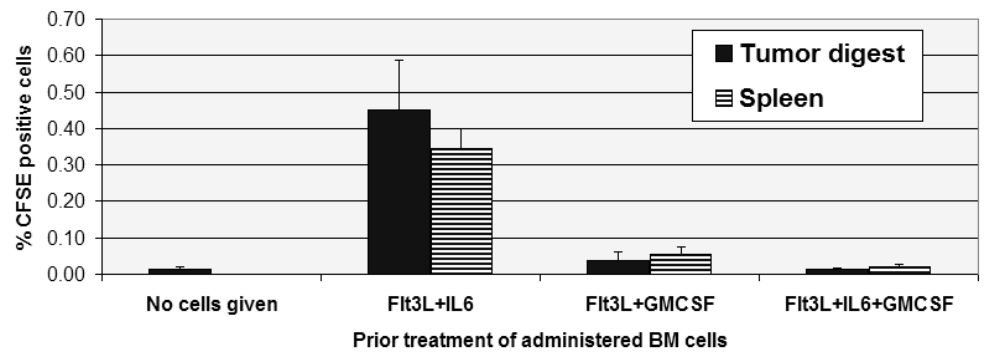

B

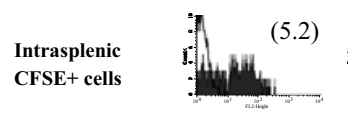

Intratumoral CFSE+ cells

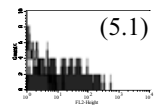

$\underline{\text { CD40 }}$ $\underline{\text { MHC Class II }}$
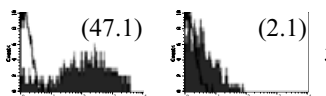

$\underline{B 7.1}$
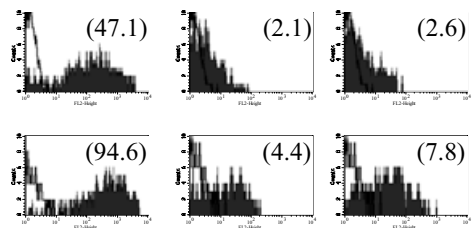

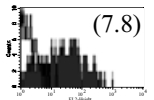

$\underline{B} 7.2$

$\underline{\text { Ox40L }}$
Figure 5. In vivo fate of $\mathrm{BM}$ cells administered following step 1 conditioning treatments. Eighteen million BM cells were labelled with CFSE and given by tail vein to mice bearing 10-day MCA-203 subcutaneous tumors. Forty-eight hours later, mice were euthanized and single cell suspensions of tumors and spleens processed for FACS analyses. (A) Frequency of CFSE ${ }^{+}$ cells recovered in tumor or spleen. Each bar shows average of 5 synchronously analyzed tumors or spleens $( \pm \mathrm{SD})$ in a single experiment for each step 1 condition or for background staining (no cells given). Flt3L + IL-6 versus Flt3L + GMCSF or Flt3L + IL-6 + GMCSF conditioning, $P<.001$. (B) Phenotype of $\mathrm{CFSE}^{+}$cells recovered in tumor or spleen following in vitro Flt3L + IL-6 conditioning. Numbers in parentheses in each histogram display mean fluorescence specificity indices. Results in panels A and B are representative of 3 separate experiments.

\section{Discussion}

Certain timely stimuli can condition DCs to adhere to an extended period of programming. For example, we recently reported that exposure of monocyte-derived human DCs to IFN $\gamma+$ LPS not only stimulated an initial burst of IL12p70 secretion, but also licensed a second burst of IL12p70 secretion even days later if CD40 ligation was experienced. ${ }^{6}$

We report here that durable DC programming can also be secured at remarkably early stages of hematopoiesis, even prior to discernible phenotypic DC lineage commitment. Exposure of fresh mouse BM to Flt3L + IL-6 triggered multilog expansion of CD34pos progenitor cells, and committed nearly all cells to subsequent DC differentiation. Such programming included subsequent spontaneous up-regulation of $\mathrm{MHC} /$ costimulatory molecules, as well as nearly uniform responsiveness to DC1 polarization stimuli. Moreover, proliferative conditioning with Flt3L + IL-6 conferred progressive resistance to many tumorassociated immunosuppressive factors, as well as the capacity to respond to either tumor contact or to TGF $\beta$ with facilitated DC1 polarization.

Flt3L + IL-6 treatment produced identical licensing whether performed upon unfractionated BM or upon sorted CD34pos cells, indicating that more primitive $\left(\mathrm{CD} 34^{\mathrm{neg}} / \mathrm{Sca}-1^{\text {pos }}\right)$ hematopoietic stem cells were not essential conditioning targets. Pronounced biasing toward ultimate DC differentiation was observed whether step 1 conditioning was performed in Flt3L + IL-6 or Flt3L alone. However, in the absence of coconditioning by IL-6, Flt3L-induced DC precursors remained scant in number and displayed early maturation, whereas the inclusion of IL-6 produced an immense proliferative pool of $\mathrm{CD} 11 \mathrm{c}^{\text {neg }} / \mathrm{MHC}$ class II $^{\text {neg }}$ cells that were nonetheless already committed to subsequent DC differentiation. Such still phenotypically undifferentiated cells displayed the capacity both for wide distribution following intravenous injection and for spontaneous DC differentiation/maturation after entry into tumor.

IL-6 and its receptor transducing component gp130 have long been recognized to synergize for stem cell proliferation with receptor tyrosine kinase-activating stimuli, including both c-kit ligand (SCF) and Flt3-L, ${ }^{18-20}$ but only combined Flt3L + IL-6 also preconditioned for nearly global differentiation of expanded CD34pos cells into spontane- ously maturing DCs. Paradoxically, although GMCSF also synergized with Flt3L to induce step 1 stem cell proliferation, GMCSF antagonized every DC-licensing effect attributable to Flt3L.

Our evidence supports the existence of at least 2 discrete pathways for conventional DC differentiation with strikingly divergent functional outcomes, a Flt3L-promoted STAT3dependent pathway and a GMCSF-promoted STAT5-dependent pathway (schematized in Figure 7). The Flt3L-promoted pathway was highly potentiated by IL-6, most likely due to the latter's capacity to confer sustained STAT3 activation during extended CD34 ${ }^{\text {pos }}$ cell proliferation, and biased for differentiation into DC precursors that were uniformly facilitated for DC1 polarization, spontaneous DC maturation, and activation by tumor contact. In contrast, the GMCSF-promoted, STAT5dependent pathway gave rise to diverse myeloid elements, including DCs that failed to mature in the absence of further downstream driving signals such as TLR agonists.

We hypothesize that STAT5-dependent DC differentiation constitutes the normally dominant pathway, based on several observations: (1) the inclusion of GMCSF during CD34pos progenitor cell proliferation blocked STAT3 activation and promoted STAT5-dependent myeloid differentiation regardless of whether Flt3L or Flt3L + IL-6 were also present; (2) recent studies indicate that STAT3-dependent DC differentiation is not normally detectable in adult mice. ${ }^{42}$ Nonetheless, we have observed that BM even from much older mice remains highly responsive to exogenous Flt3L or Flt3L + IL-6 conditioning (not shown). STAT3-dependent preempting of normal hematopoiesis in favor of dedicated DC production may therefore reflect a reserved host response, perhaps to life-threatening infection of the BM itself. Consistent with this possibility, Flt3L and IL-6 are strongly induced by myelosuppression and BM inflammation, respectively. ${ }^{43,44}$

Despite the competing roles played by STAT3 and STAT5 in DC differentiation, it is also apparent that their respective impacts change before and after DC differentiation occurs. Pronounced sequential STAT3/STAT5 activation (ie, Flt3L + IL6-conditioned DCs in Figure 6A) corresponded to the greatest observed attainment of DC tumor competence, and neither STAT3 nor STAT5 knockout BM responded optimally to Flt3L + IL-6 conditioning. The preemptive impacts of GM-CSF 
A

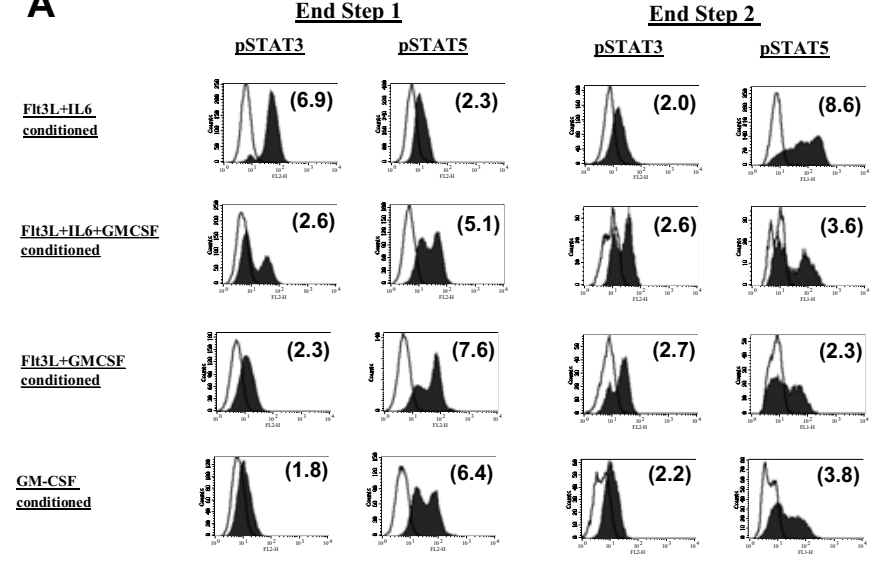

B

Stat 5 ko vs. wildtype littermate

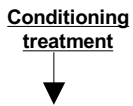

St5 LM,

Ft3L+IL6
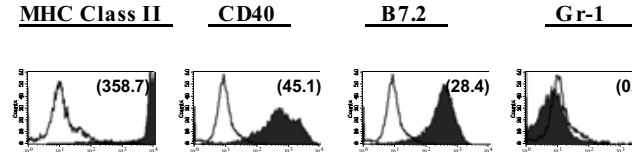

St5 KO,

FIt3L+ IL6
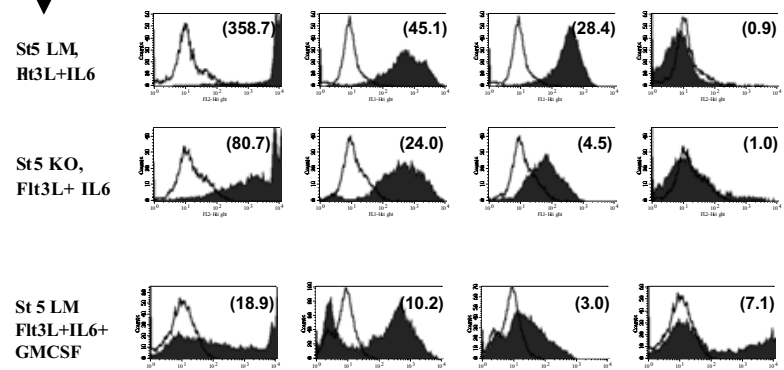

St5 KO,

Ht3L+IL6+

GMCSF
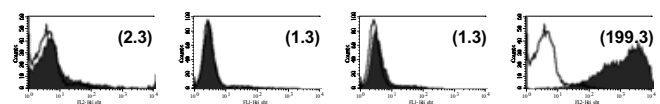

Figure 6. Impacts of STAT3 and STAT5 on BM conditioning. (A) Representative intracellular staining for pSTAT3 (pY705) versus pSTAT5 ( $p$ Y694) following various step 1 conditioning regimens, either at end of 6-day step 1 culture (left) or after an additional 48-hour step 2 culture in GMCSF + IL-4 (right). Numbers in parentheses in each histogram display mean fluorescence specificity indices. Results are representative of 4 (left) and 3 (right) separate experiments, respectively. (B-D) Capacities of STAT5 or STAT3 knockout mice to respond to various conditioning regimens. (B) BM was prepared from STAT5 ${ }^{\mathrm{KO}}$ mice (St5 KO) or from littermates expressing wildtype STAT5 (St5 LM) and subjected to step 1 conditioning with Ft3L+IL-6 or Flt3L+IL-6+GMCSF. Results shown for Flt3L+IL-6+GMCSF are also highly representative of GMCSF or Flt3L+IL-6+GMCSF conditioning outcomes (not shown). Proliferation kinetics during step 1 culture were comparable for KOs versus LMs (not shown). Histograms display mean fluorescence specificity indices for DC maturation markers following 48 hours of step 2 culture in GMCSF+IL-4, with CpG + LPS added during the final 18 hours. Results shown are representative of 3 experiments. (C) BM was prepared from STAT3KO mice ("St3 KO") or from LMs expressing wild-type STAT3 ("St3 LM") and subjected to step 1 conditioning and step 2 culture as in panel A. Results shown for Flt3L+IL-6+GMCSF are also highly representative of GMCSF or Flt3L+GMCSF conditioning. Proliferation kinetics during step 1 culture were comparable for KOs versus LMs, except that abject culture failure occurred when "St3 KO" BM was conditioned with Flt3L+ IL-6 (not shown). Results shown are representative of 2 experiments. (D) ELISA comparisons of supernatants for IL12p70 at end of step 2 cultures. BM was obtained from KO mice or from matched wildtype LMs, placed in standard step 1 cultures with Flt3L+IL-6, Flt3L+IL-6+GMCSF, or GMCSF alone as conditioning agents, and finally placed in 48-hour step 2 cultures with GMCSF+IL-4, and CpG+LPS added during the final 18 hours. Culture failure was observed only when STAT3 ${ }^{\mathrm{KO}}$ BM was conditioned with FIt3L + IL-6. Data are presented as means plus or minus SD of 3 replicates and are representative of 3 (STAT5 ${ }^{\mathrm{KO}}$ vs LM) or 2 (STAT3 ${ }^{\mathrm{KO}}$ vs LM) independently performed experiments.

during step 1 of culture appear to be mediated through early combined STAT3 inhibition and STAT5 activation (Figure 6). Paradoxically, however, appropriately delayed exposure to GMCSF may promote maximal DC1 polarization through the identical STAT modulations (Figure 7).

The mechanism(s) causing Flt3L + IL-6 preconditioned DCs to respond to tumor as a maturational signal remains to be elucidated. A plausible mechanism involves DC activation via enhanced expression of lectin receptors such as asialoglycoprotein receptor and DEC-205 (Figure S3C), ${ }^{3,45}$ since identical or similar carbohydrate receptors are used by tumoricidal macrophages to bind and kill tumor cells in an MHC-unrestricted and antigen-unrestricted manner. ${ }^{46,47}$ Many tumor cells bind lectin more avidly than nontransformed cells, due to a chronically high density of exposed carbohydrates and a diminished presence of differentiation elements that normally mask such carbohydrates. ${ }^{46,47}$ Similarly, we have observed that exposure to albumin cross-linked with mannose or $N$-acetyl-glucosamine accelerates the maturation of Flt3L + IL-6-conditioned BM cells (not shown).

We are investigating why most proliferative conditioning treatments caused DC preparations to be highly toxic to T-cell cultures. Such lethality was unlikely attributable to activation of regulatory $\mathrm{T}$ cells or indoleamine dioxygenase-expressing plasmacytoid DCs (pDCs), since toxicity was not remedied by adding exogenous IL-2 to the T-cell cocultures. ${ }^{48,49}$ There was, 


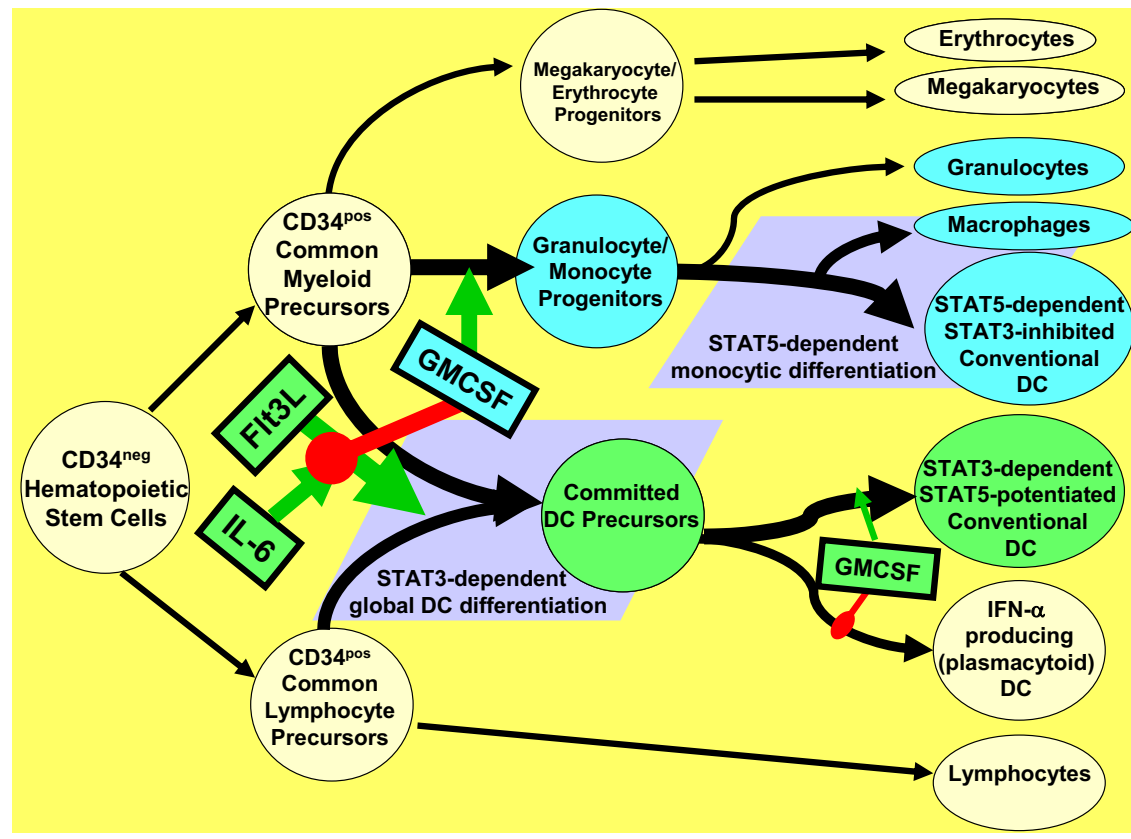

Figure 7. Schematic representation of postulated STAT-dependent DC differentiation pathways. Flt3L stimulates pan-differentiation of CD34pos common myeloid and common lymphocyte precursors into CD11 ${ }^{+}$ committed DC precursors via a STAT3-dependent process. This is markedly potentiated by IL-6, but is dominantly suppressed by step 1 exposure to GMCSF, due to inhibition of STAT3 activation and concomitant STAT5 activation (Figure 6A). Such step 1 GMCSF exposure instead favors differentiation of $\mathrm{CD} 34^{+}$common myeloid precursors into granulocyte/monocyte progenitors (rather than committed DC precursors). The granulocyte/ monocyte progenitors achieve subsequent multilineage differentiation, including STAT5-dependent differentiation into conventional DCs and macrophages, and STAT5independent differentiation into neutrophils (Figure 6B) Phenotypically conventional DCs generated by STAT3versus STAT5-dependent pathways differ in many critical characteristics (Figures 1-5). It should be emphasized that although step 1 exposure to GMCSF blocks STAT3 dependent DC differentiation, step 2 exposure of committed DC precursors to GMCSF may instead promote maturation and DC1-polarization, again by stimulating STAT5 and inhibiting STAT3 (Figure 6A)

however, a correlation between the Gr-1 ${ }^{\text {pos }}$ cell-inducing tendency of individual conditioning treatments and observed lethality. It is therefore possible that the Gr-1 ${ }^{\text {pos }}$ subpopulation(s) mediate the untoward effects of many of the conditioning treatments. ${ }^{35,36}$ Consistent with this possibility, heightened induction of $\mathrm{Gr}^{\text {pos }}$ myeloid suppressor cells appears to be the basis of immune disruption by high GMCSF-producing vaccine formulations. $^{36}$ In contrast to GMCSF-conditioned cultures, Flt3L + IL-6-conditioned DC cultures were exceptional for their low Gr $1^{\text {pos }}$ content and consistent absence of toxicity, even when added to $\mathrm{T}$ cells in high proportions.

The above experiments, as well as preliminary studies with human CD34pos progenitor cells (not shown), demonstrate that Flt3L + IL-6 may provide an effective means to proliferate, condition, and mobilize highly therapeutic DC precursors for tumor therapy. It has long been appreciated that IL-6 has extremely potent therapeutic properties against established mouse tumors, even when administered as a single agent. ${ }^{50}$ We postulate that the major mechanistic role and benefit of IL-6 therapy will be in tandem with Flt3L to proliferate and condition tumor-competent DC precursor populations in cancer patients.

\section{Acknowledgments}

We thank Dr Walter Storkus for his extremely helpful suggestions.

This work is supported by National Institutes of Health (NIH, Bethesda, MD) grants R01-CA089511, R01-CA103946, and RO1CA129815.

\section{Authorship}

Contribution: P.A.C. wrote the paper, designed the experiments, performed experiments, and analyzed data; G.K.K., S.S., B.J.C., J.F.T., K.D.B., X.-Y.F., and C.S.C. participated in experimental design and data analyses; and Z.W., W.-J.Z., M.A., C.A.D., H.N., C.C.P., and T.D.J. participated in experimental performance and data analyses.

Conflict-of-interest disclosure: The authors declare no competing financial interests.

Correspondence: Peter A. Cohen, Center for Surgery Research, NE6, Cleveland Clinic Foundation, 9500 Euclid Avenue, Cleveland, OH 44195; e-mail: cohenp@ccf.org.

\section{References}

1. Rosenberg SA, Yang JC, Restifo NP. Cancer immunotherapy: moving beyond current vaccines. Nat Med. 2004;10:909-915.

2. Banchereau J, Palucka AK, Dhodapkar M, et al. Immune and clinical responses in patients with metastatic melanoma to CD34(+) progenitorderived dendritic cell vaccine. Cancer Res. 2001; 61:6451-6458.

3. Bonifaz L, Bonnyay D, Mahnke K, et al. Efficient targeting of protein antigen to the dendritic cell receptor DEC-205 in the steady state leads to antigen presentation on major histocompatibility complex class I products and peripheral CD8 + T cell tolerance. J Exp Med. 2002;196:1627-1638

4. Xu S, Koski GK, Faries M, et al. Rapid high efficiency sensitization of CD8 + T cells to tumor antigens by dendritic cells leads to enhanced func- tional avidity and direct tumor recognition through an IL-12 dependent mechanism. J Immunol. 2003:171:2251-2261.

5. Berhanu A, Huang J, Alber SM, Watkins SC, Storkus WJ. Combinational FLt3 ligand and granulocyte macrophage colony-stimulating factor treatment promotes enhanced tumor infiltration by dendritic cells and antitumor CD8(+) T-cell cross-priming but is ineffective as a therapy. Cancer Res. 2006;66:4895-4903.

6. Czerniecki BJ, Koski GK, Koldovsky U, et al. Targeting HER2-neu in early breast cancer development using dendritic cells with staged interleukin-12 burst secretion. Cancer Res. 2007;67:1842-1852.

7. Napolitani G, Rinaldi A, Bertoni F, Sallusto F, Lanzavecchia A. Selected Toll-like receptor agonist combinations synergistically trigger a $T$ helper type 1-polarizing program in dendritic cells. Nat Immunol. 2005;6:769-776.

8. Czerniecki BJ, Carter C, Rivoltini L, et al. Calcium ionophore treated peripheral blood monocytes and dendritic cells rapidly display characteristics of activated dendritic cells. J Immunol. 1997;159: 3823-3837.

9. Mailliard RB, Wankowicz-Kalinska A, Cai Q, et al. alpha-type-1 polarized dendritic cells: a novel immunization tool with optimized CTL-inducing activity. Cancer Res. 2004;64:5934-5937.

10. Gajewski TF, Renauld JC, Van Pel A, Boon T. Costimulation with B7-1, IL-6, and IL-12 is sufficient for primary generation of murine antitumor cytolytic T lymphocytes in vitro. J Immunol. 1995;154: 5637-5648.

11. Avigan $\mathrm{D}, \mathrm{Wu} Z$, Gong J, et al. Selective in vivo mobilization with granulocyte macrophage 
colony-stimulating factor (GM-CSF)/granulocyteCSF as compared to G-CSF alone of dendritic cell progenitors from peripheral blood progenitor cells in patients with advanced breast cancer undergoing autologous transplantation. Clin Cancer Res. 1999;5:2735-2741.

12. Pulendran B, Banchereau J, Burkeholder S, et al. Flt3-ligand and granulocyte colony-stimulating factor mobilize distinct human dendritic cell subsets in vivo. J Immunol. 2000;165:566-572.

13. Parajuli P, Mosley RL, Pisarev V, et al. Flt3 ligand and granulocyte-macrophage colony-stimulating factor preferentially expand and stimulate different dendritic and T-cell subsets. Exp Hematol. 2001;29:1185-1193.

14. Kobie JJ, Wu RS, Kurt RA, et al. Transforming growth factor beta inhibits the antigen-presenting functions and antitumor activity of dendritic cell vaccines. Cancer Res. 2003;63:1860-1864.

15. Gabrilovich D, Ishida T, Oyama T, et al. Vascular endothelial growth factor inhibits the development of dendritic cells and dramatically affects the differentiation of multiple hematopoietic lineages in vivo. Blood. 1998;92:4150-4166.

16. Yang AS, Lattime EC. Tumor-induced interleukin 10 suppresses the ability of splenic dendritic cells to stimulate CD4 and CD8 T-cell responses. Cancer Res. 2003;63:2150-2157.

17. Kaliński P, Schuitemaker JH, Hilkens CM, Kapsenberg ML. Prostaglandin E2 induces the final maturation of IL-12-deficient CD1a+CD83+ dendritic cells: the levels of IL-12 are determined during the final dendritic cell maturation and are resistant to further modulation. J Immunol. 1998; 161:2804-2809.

18. Ebihara $Y$, Tsuji $\mathrm{K}$, Lyman SD, et al. Synergistic action of Flt3 and gp130 signalings in human hematopoiesis. Blood. 1997;90:4363-4368.

19. Sui X, Tsuji K, Tanaka R, et al. gp130 and c-Kit signalings synergize for ex vivo expansion of human primitive hemopoietic progenitor cells. Proc Natl Acad Sci U S A. 1995;92:2859-2863.

20. Brasel K, McKenna HJ, Charrier K, et al. Flt3 ligand synergizes with granulocyte-macrophage colony-stimulating factor or granulocyte colonystimulating factor to mobilize hematopoietic progenitor cells into the peripheral blood of mice. Blood. 1997;90:3781-3788.

21. Peng L, Kjaergaard J, Plautz GE, et al. Tumorinduced Lselectin ${ }^{\text {high }}$ suppressor $T$ cells mediate potent effector $\mathrm{T}$ cell blockade and cause failure of otherwise curative adoptive immunotherapy. J Immunol. 2002;169:4811-4821.

22. Parkhurst MR, DePan C, Riley JP, Rosenberg SA, Shu S. Hybrids of dendritic cells and tumor cells generated by electrofusion simultaneously present immunodominant epitopes from multiple human tumor-associated antigens in the context of MHC class I and class II molecules. J Immunol. 2003; 170:5317-5325.

23. Teglund S, McKay C, Schuetz E, et al. Stat5a and Stat5b proteins have essential and nonessential, or redundant, roles in cytokine responses. Cell. 1998;93:841-850.

24. Cui Y, Riedlinger G, Miyoshi K, et al. Inactivation of Stat5 in mouse mammary epithelium during pregnancy reveals distinct functions in cell proliferation, survival, and differentiation. Mol Cell Biol. 2004;24:8037-8047.

25. Bunting KD, Bradley HL, Hawley TS, et al. Reduced lymphomyeloid repopulating activity from adult bone marrow and fetal liver of mice lacking expression of STAT5. Blood. 2002;99:479-487.

26. Welte T, Zhang SS, Wang T, et al. STAT3 deletion during hematopoiesis causes Crohn's disease-like pathogenesis and lethality: a critical role of STAT3 in innate immunity. Proc Natl Acad Sci U S A. 2003;100:18791884.

27. Kuriyama H, Shimizu K, Lee W, et al. Therapeutic vaccine generated by electrofusion of dendritic cells and tumour cells. Dev Biol (Basel). 2004;116:169-178.

28. Cohen PA, Cohen PJ, Rosenberg SA, Mule JJ. CD4+ T-cells from mice immunized to syngeneic sarcomas recognize distinct, non-shared tumor antigens. Cancer Res. 1994;54:1055-1058.

29. Peng L, Kjaergaard J, Weng DE, et al. Helperindependent CD8+/CD62L low T cells with broad anti-tumor efficacy are naturally sensitized during tumor progression. J Immunol. 2000;165:5738-5749.

30. Tamura $\mathrm{T}$, Tailor $\mathrm{P}$, Yamaoka $\mathrm{K}$, et al. IFN regulatory factor-4 and -8 govern dendritic cell subset development and their functional diversity. J Immunol. 2005;174:2573-2581.

31. Onai N, Obata-Onai A, Tussiwand R, Lanzavecchia A, Manz MG. Activation of the Flt3 signal transduction cascade rescues and enhances type I interferon-producing and dendritic cell development. J Exp Med. 2006;203:227-238.

32. Wang LX, Huang WX, Graor H, et al. Adoptive immunotherapy of cancer with polyclonal, $10^{8}$. fold hyperexpanded, CD4+ and CD8+ T cells. J Transl Med. (DOI 10.1186/1479-5876-2-41). 2004;2:41.

33. Maraskovsky E, Brasel K, Teepe M, et al. Dramatic increase in the numbers of functionally mature dendritic cells in Flt3 ligand-treated mice: multiple dendritic cell subpopulations identified. J Exp Med. 1996;184:1953-1962.

34. Brasel K, De Smedt T, Smith JL, Maliszewski CR Generation of murine dendritic cells from flt3 ligand-supplemented bone marrow cultures. Blood. 2000;96:3029-3039.

35. Kusmartsev S, Nefedova Y, Yoder D, Gabrilovich DI. Antigen-specific inhibition of CD8+ T cell response by immature myeloid cells in cancer is mediated by reactive oxygen species. J Immunol. 2004;172:989-999.

36. Serafini P, Carbley R, Noonan KA, et al. Highdose granulocyte-macrophage colony-stimulating factor-producing vaccines impair the immune response through the recruitment of myeloid suppressor cells. Cancer Res. 2004:64:6337-6343.

37. Iborra S, Abanades DR, Parody N, et al. The immunodominant T helper 2 (Th2) response elicited in BALB/c mice by the Leishmania LiP2a and $\mathrm{LiP} 2 \mathrm{~b}$ acidic ribosomal proteins cannot be reverted by strong Th1 inducers. Clin Exp Immunol. 2007;150:375-385.

38. Laouar Y, Welte T, Fu XY, Flavell RA. STAT3 is required for Flt3L-dependent dendritic cell differentiation. Immunity. 2003;19:903-912.

39. Majka M, Ratajczak J, Villaire G, et al. Thrombopoietin, but not cytokines binding to gp130 protein-coupled receptors, activates MAPKp42/44, AKT, and STAT proteins in normal human CD34+ cells, megakaryocytes, and platelets. Exp Hematol. 2002;30:751-760.

40. Lehtonen A, Matikainen S, Miettinen M, Julkunen I. Granulocyte-macrophage colony-stimulating factor (GM-CSF)-induced STAT5 activation and target-gene expression during human monocyte/ macrophage differentiation. J Leukoc Biol. 2002; 71:511-519.

41. Giron-Michel J, Fogli M, Gaggero A, et al. Detection of a functional hybrid receptor gammac/GMCSFRbeta in human hematopoietic CD34+ cells. J Exp Med. 2003;197:763-775.

42. Kortylewski M, Kujawski M, Wang T, et al. Inhibiting Stat3 signaling in the hematopoietic system elicits multicomponent antitumor immunity. Nat Med. 2005;11:1314-1321.

43. Chklovskaia E, Jansen W, Nissen C, et al. Mechanism of flt3 ligand expression in bone marrow failure: translocation from intracellular stores to the surface of $T$ lymphocytes after chemotherapy-induced suppression of hematopoiesis. Blood. 1999;93:2595-2604.

44. Gaugler MH, Squiban C, Claraz M, et al. Characterization of the response of human bone marrow endothelial cells to in vitro irradiation. $\mathrm{Br} \mathrm{J}$ Haematol. 1998;103:980-989.

45. Valladeau J, Duvert-Frances V, Pin JJ, et al. Immature human dendritic cells express asialoglycoprotein receptor isoforms for efficient receptormediated endocytosis. J Immunol. 2001;167: 5767-5774.

46. Cohen PA, Peng L, Plautz GE, et al. CD4+ T cells in adoptive immunotherapy and the indirect mechanism of tumor rejection. Crit Rev Immunol. 2000;20:17-56.

47. Oda S, Sato M, Toyoshima S, Osawa T. Binding of activated macrophages to tumor cells through a macrophage lectin and its role in macrophage tumoricidal activity. J Biochem (Tokyo). 1989;105: 1040-1043.

48. Powell DJ Jr, Parker LL, Rosenberg SA. Largescale depletion of CD25+ regulatory $T$ cells from patient leukapheresis samples. J Immunother. 2005;28:403-411.

49. Munn DH, Sharma MD, Hou D, et al. Expression of indoleamine 2,3-dioxygenase by plasmacytoid dendritic cells in tumor-draining lymph nodes. J Clin Invest. 2004;114:280-290.

50. Mulé JJ, Custer MC, Travis WD, Rosenberg SA. Cellular mechanisms of the antitumor activity of recombinant IL-6 in mice. J Immunol. 1992;148:2622-2629. 